414. $\mathrm{Z}$ i $\mathrm{m} m$ e $\mathrm{r} \mathrm{m}$ a $\mathrm{n} \mathrm{n}$, R., und $\mathrm{H}$ e y d e r, R., Brutnotizen zur Rochlitzer Ornis 1906. Zeitschr. f. Oolog. u. Orn. 16. Jg. (1906/07) p. 105-110, 124-127, 135-139.

415. - Brutnotizen zur Rochlitzer Ornis 1908. Ebenda 19. Jg. (1909/10) p. 10-12, 24-26.

\title{
III.
}

\section{Die geographische Verbreitung der Vögel im Königreich Sachsen.}

\section{Allgemeiner Teil.}

Von vornherein war für die Art und Weise der von mir gepflogenen faunistischen Landesaufnahme vor allem der Gedanke bestimmend, den Umfang des g e g e n w ä r t i g e n Bestandes der Brutarten, also des eigentlichen faunenbildenden Elements, nach Möglichkeit genau aufzunehmen. Dafs mir das nur in weit bescheidenerem Mafse, als ich dies anstrebte, gelang, empfindet niemand besser als ich, und ich bitte in dem etwas hochtrabenden Titel, den ich dieser Arbeit voransetzte, nicht mehr als ein bequemes Zitat zu sehen, als zutreffende Aufschrift aber den Untertitel betrachten zu wollen. Ich schrieb die Arbeit rein unter dem Gesichtswinkel der geographischen Verbreitung, mufste auch unter Hinblick auf den relativ knappen Raum auf die Wiedergabe biologischer Einzelheiten verzichten und konnte nur gelegentlich gewisse lokale biologische Eigenheiten streifen. Ein nachahmenswertes Vorbild für meine Aufgabe erblickte ich in William B a ers Abhandlung ,Zur Ornis der preufsischen Oberlausitz", in welcher dieser Schriftsteller mit grofser Sorgfalt und ungewöhnlich treffender Form das für den Aufenthalt einer jeden Art charakteristische Landschaftsgepräge hervorzuheben versteht, was seine Arbeit weit über den Rahmen gleichartiger Veröffentlichungen erhebt. Freilich scheinen in seinem Gebiete die Grenzen zwischen den einzelnen Boden- und Vegetationsgemeinschaften weit schärfer zutage $\mathrm{zu}$ treten als beispielsweise in vielen Teilen Sachsens, wo sie vielfach verschwimmen und unmerklich ineinander übergehen. Dafür aber zeichnet unser Gebiet eine ganz eigenartige, in tiergeographischer Beziehung hochbedeutsame Bodengestaltung aus. Im Süden erstreckt sich längs der böhmischen Grenze ein bedeutender Gebirgswall, das Erzgebirge, westlich anschliefsend das Elstergebirge, im Osten das Elbsandstein-, Lausitzer und Zittauer Gebirge, für die Flüsse mit Ausnahme der Elbe, die das Elbsandsteingebirge durchbricht, die Wasserscheide. Nach Norden zu dacht sich das ganze Gebiet allmählig ab, sodals es in seiner Ausdehnung von Süd nach Nord Höhenlagen von über $1200 \mathrm{~m}$ bis unter $100 \mathrm{~m}$ Seehöhe durchläuft und damit zwingt, nicht nur die horizontale, sondern auch die 
vertikale Verbreitung der Vogelarten zu untersuchen. Nur mit seinem nördlichen Teil reicht das Königreich hinüber in die Norddeutsche Tiefebene, in das spezielle Verbreitungsgebiet der Kiefer (Pinus silvestris) als waldbildender Baum, während der weitaus gröfste Teil vom Mitteldeutschen Bergund Hügelland beherrscht wird, dessen Charakterbaum die von der moderuen Forstwirtschaft auch in die Ebene getragene Fichte (Picea excelsa) ist. Betrachten wir noch weiter die Vegetationsverhältnisse des eigentlichen Gebirges, soweit sie für die Zusammensetzung der Vogelwelt einschneidende Wirkung haben, so ist es eine einzige Laubholzart, die hier eine wirkliche Bedeutung gewinnt, die Rotbuche (Fagus silvatica), die bis $\mathrm{zu}$ Höhen von $860 \mathrm{~m}$ in reinen, z. T. sehr alten Beständen gröfsere Flächen bedeckt. Neben ihr verdient als Nahrungspflanze die vielfach längs der Landstrafsen angepflanzte Eberesche (Sorbus aucuparia) genannt $\mathrm{zu}$ werden. Alle übrigen Laubhölzer besitzen auf die Zusammensetzung dieser Wälder keinen nennenswerten Einflufs, auch Tanne und Kiefer sind verhältnismälsig einzeln, der Wachholder selten. Stellenweise finden sich in den höheren Lagen Hochmoore mit partiell heideartigem Charakter. Edlere Obstsorten gedeihen von $700 \mathrm{~m}$ an nur noch mit Beschwerden, über $800 \mathrm{~m}$ überhaupt nicht mehr. Feldbau steigt bis über $1000 \mathrm{~m}$ an, ist aber schon $200 \mathrm{~m}$ tiefer dürftig, und die Wiesen überzieht ein mattenartiger, niedriger Bergrasen. Die wenigen stehenden Gewässer sind tiefgründig, ihnen fehlt deshalb der breite Vegetationsgürtel der Tieflandsteiche, deren bezeichnendster Pflanzenwuchs, das Phragmitetum, schon bei $500 \mathrm{~m}$ stark zurücktritt, weiter hinauf aber ganz verschwindet und einer aus Cyperaceen, Juncaceen, Schachtelhalmen und verschiedenen Gräsern zusammengesetzten Teichflora Platz macht. Stellen wir der ebengeschilderten Landtschaftsform ihr Extrem gegenüber, die gelinde, niederscblagärmere Niederung, der Boden jungen und jüngsten geologischen Schichten angehörend, mit ihren fruchtbaren Ackerländereien und üppigen Wiesenfluren, ihren strauchwerkreichen Feldhölzern und schilfrohrbewaldeten 'Teichen, längs der flachen Flufstäler den aus den mannigfachsten Laubhölzern gebildeten, eine artenreiche Unterflora beherbergenden Auewald hervorbringend, östlich der Elbe auf grofse Strecken hin Kiefernsandheiden aufweisend, so tun sich uns schärfer, als wir infolge des steten Ineinanderfliefsens beider Gebiete in den hügeligen Grenzgegenden zu sehen gewohnt sind, die Unterschiede dar, welche die im folgenden Abschnitt gewürdigte $\mathrm{Höh}$ enschichtung der Vogelwelt bewirken. Wohl fast ausschliefslich ist diese Höhengliederung von dem Vorkommen oder Fehlen der von der jeweiligen Vogelart bevorzugten Landschaftsform bezw. Pflanzenwelt abbängig und wird nur in gewissen Fällen (bei Überwinterung) direkt von den klimatischen Unterschieden beeinflufst. Nimmt doch im allgemeinen die 
Jahrestemperatur auf je $100 \mathrm{~m}$ Steigung um nur $0,57^{\circ} \mathrm{ab}$. Macht sich sonach für die Vögel während der Fortpflanzung das Gebirge einschneidend bemerkbar, so könute für die Zugvögel eine ähnliche Wirkung erwartet und angenommen werden, dafs die wandernden Scharen ein Überfliegen der Höhenzüge vermeiden. Das trifft jedoch nicht zu, wie mir verschiedene Beispiele beweisen. So flog vor einiger Zeit an dem Unterkunftshaus auf dem Fichtelberge ein Exemplar Gallinula chloropus an, und im nahen Gottesgab beobachtete man durchziehende Störche, auch wurde am dortigen Moor 1 Totanus pugnax geschossen. Anders liegt jedoch die Frage hinsichtlich der Besiedelung der höher gelegenen Brutgebiete durch die zurückkommenden Vögel. Es ist hier nicht von der Hand $\mathrm{zu}$ weisen, dafs bei dem Umstand, dafs in unserem engeren Gebiet die nördlich gelegenen Striche die gelindesten und die südlichen, von den Zugvögeln zuerst zu erreichenden zugleich die unwirtlichsten sind, ein Überfliegen der Brutplätze und ein allmählig erfolgendes Aufwärtsgehen längs der Täler und damit eine Rückwärtsbewegung die Regel sein kann, besonders für die zeitig eintreffenden Arten (Stare, Lerchen, Bachstelzen etc.), die sehr unter den Unbilden der Witterung zu leiden haben. Die von Meyer und Helm gesammelten Zugdaten lassen hier freilich völlig im Stich; sie resultieren wohl zu sehr aus Gelegenheitsbeobachtungen. Ich möchte bei dieser Gelegenheit noch ein Wort einschalten über die vermeintliche Bedeutung der Flufsläufe als Zugstrafsen. Es bestätigt sich nach dem von mir durchgearbeiteten Datenmaterial und meinen eigenen Erfahrungen nicht, dafs manche Arten von Wasservögeln beim Zug generell den Flufsläufen folgen. Es ergibt sich vielmehr das Gegenteil, welches sich ganz besonders an den nächtlich wandernden und dabei rufenden Arten (z. B. Ardea cinerea, Tringoides, Numenius, Charadrius apricarius u. s. w.) feststellen läfst. Zur Rast fallen sie allerdings an nahrungversprechenden, zutällig in der Flugbahn liegenden Gewässern ein und werden hier erlegt oder auf andere Weise festgestellt, wodurch sich die eben erwähnte irrtümliche Anschauung bilden konnte. -

Werfen wir noch rasch einen Blick den Schicksalen zu, die der Vogelwelt unseres übervölkerten Landes in der nächsten Zeit bevorstehen, so läfst sich im Gefühl völliger Ohnmacht nur konstatieren, dafs wie in allen dicht bewohnten Staaten auch bei uns die verderblichen Einflüsse der Kultur ihre Wirkung getan haben und in Zukunft weiter tun werden. Fischreiher, Schwarzstorch, die Milane, Fischadler, Uhu und Kolkrabe, all' die prächtigen Vogelgestalten sind verschwunden, und Storch und Rohrdommel, Haselhuhn und Wanderfalk, Blaurake und Nachtigall, sie werden folgen. Wir können ihnen Galgenfristen erwirken, den Gang der Dinge aufzuhalten vermögen wir nicht! -

Was die allgemeine zoogeographische Struktur unseres Gebietes anlangt, so heimatet hier, wie schon nach der durch 
das Gebiet verlaufenden Grenzzone in der Verbreitung von Corvus corone und cornix vermutet werden kann, eine in jeder Beziehung rein zentraleuropäische Vogelwelt, die selbst jenen leisen östlichen Einschlag, der unsere Nachbarprovinz Preufsisch-Schlesien auszeichnet (Brüten von Sprosser, Gänsesäger, häufigeres Brüten von Moorente, Nachtreiher, Abendfalk u. s. w.), so ziemlich vermissen lälst. Erwähnenswert bleiben höchstens noch für unsere Gebirge, die nirgends die Baumgrenze erreichen, die noch vorhandenen Spuren einer „Relikten-Ornis“, als welche Alpenamsel, Tannenhäher und ev. auch der noch sicher nachzuweisende Alpenleinfink $\mathrm{zu}$ betrachten sind.

Die Vogelwelt unserer Grenzgebiete, die unter Vergleich mit der sächsischen Ornis zu weiteren tiergeographischen Schlüssen führen würde, ist zusammenfassend gegenwärtig sehr ungleich bearbeitet. Schlesien besitzt eine schöne Arbeit in P. Kollibays „Vögel der Preufs. Provinz Schlesien" (1906), Böhmen eine etwas veraltete Bearbeitung in A. Fritsch's ,Wirbeltiere Böhmens" (1872), Bayern eine gute Abhandlung von A. J. Jäckel „Systemat. Übersicht der Vögel Bayerns" (1891); für Thüringen und die Provinz Sachsen fehlen entsprechende Schriften.

Zum Schlufs noch einige allgemeine Bemerkungen über diese Arbeit. In der Nomenklatur folgte ich im wesentlichen A. Reichenows „Kennzeichen der Vögel Deutschlands“ (Neudamm 1902), benannte aber diejenigen Formen, die mehrere geographische Vertreter im Gebiet aufweisen, sowio solche, die subspezifisch von der zuerst beschriebenen, aber nicht für das Gebiet nachgewiesenen sogen. Stammform getrennt sind, trinär. Umgekehrt erblickte ich in den beiden Certhiaformen Arten und benannte sie demzufolge binär. Von der Benutzung des neuen Hartertschen Werkes sah ich ab, weil dasselbe noch nicht vollständig erschienen ist und im übrigen Reichenows Handbuch für die Klärung der geographischen Beziehungen unserer sächsischen Vogelwelt völlig genügt. Ich habe ferner im allgemeinen davon Abstand nehmen müssen, das Auftreten der Arten graduell wiederzugehen; es war dies nur bei den Gastvögeln möglich. Angehörige fremder Faunengebiete ( $\mathrm{c}$. B. Lampronessa galericulata; vergl. hierzu auch Hesse, Journ. f. Ornith. 1908 p. 280) habe ich garnicht berücksichtigt. Die Zugzeiten, die infolge der verschiedensten Ursachen mancherlei Schwankungen ausgesetzt sind, gab ich auf Grund zahlreicher Daten im normalen Verlauf; Regelwidrigkeiten sind gerade hier besonders häufig. - Die Wiedergabe von Brutnotizen unterliefs ich aus Mangel an Raum.

Zum Schlusse aber möchte ich allen, die meine Bemühungen in irgendeiner Weise förderten, auch hier meinen wärmsten Dank aussprechen. Er gilt in besonderem Mafse Herrn Prof. Dr. J a c o bi, Direktor des Kgl. Zoolog.-Anthropolog. Museums in Dresden und Herrn Willi a m B a e r, Wissenschaftl. Assistent am Zoolog. Institut der Kgl. Forstakademie Tharandt, die mir die Durchsicht 
der ihnen unterstellten Sammlungen ermöglichten. Ferner förderten durch wertvolle Beiträge faunistischen Inhalts oder literarische Unterstützung die Herren Prof. Dr. He n n i c ke, Gera, Prof. M. H ö p f n e r, Rochlitz, Prof. Dr. Vo ig t, Leipzig, Dr. He s se, Berlin, Lehrer S c h l e g e l, Leipzig, Lehrer N e s t l e r, Leipzig, Kantor em. Marx, Zöschau und Lehrer Weifs m a n el, Rauschwitz (Lausitz) neben vielen anderen meine Arbeit. Ihre Unterstützung gereichte in gleichem Mafse der Sache zum Vorteil und mir zur Freude.

Damit übergebe ich diese Abhandlung der Öffentlichkeit. Man vergegenwärtige sich die Schwierigkeiten, die der Arbeitskraft des einzelnen entgegenstehen und bedenke, dafs ich einen grofsen Teil der Vorarbeiten als in Zeit und Mitteln beschränkter Industriearbeiter erledigte und erst im Laufe der letzten Jahre etwas mehr Mufse zur Verwirklichung meines Zieles fand.

\section{Besonderer Teil.}

\section{Alca torda L.}

Das einzige sächsische Belegstück des Tordalk besitzt Dr. Mushacke in Freiberg. Es ist im März 1891 bei Pegau erlegt. Nach den Angaben seines Besitzers trägt der Vogel noch das Winterkleid, die weifse Linie von der Stirn zum Auge fehlt noch, und die Querfurchen am Schnabel sind ganz leicht angedeutet.

\section{Urinator imber (Gunn.).}

Colymbus glacialis Linn. - Meyer und Helm, Verzeichnis der bis jetzt im Königreich Sachsen beobachteten Vögel (1892) p. 124.

Der Eistaucher ist sehr seltener Gast, jedenfalls weit seltener als seine Verwandten. Soweit ich dies feststellen konnte, sind nur junge Herbstvögel vorgekommen; über Alte fehlt bis jetzt jeder Nachweis. J. G. Neumann verzeichnet ihn 1828 als bei Zittau vorgekommen. Reichenbach bemerkt, dafs er jung auf die Elbe komme und dafs sich im Dresderer Museum jüngere Vögel aus der Dresdener Gegend befänden, 1820 seien 3 bei Rotschönberg (bei Nossen) erschlagen worden. E. J. J. Meyer, der Reichenbach fast stets nachschreibt, sagt wie dieser "kommt jung auf die Elbe". Held kannte ein Exemplar, das im Herbst 1862 bei Hirschfelde (bei Zittau) erlegt worden war. Im Zoolog. Museum in Dresden befindet sich ein juv. älterer Herkunft mit der Bezeichnung „Sachsen“, im Zoolog. Institut Leipzig nach Hesse ein $\sigma^{7}$ juv. vom 17. XI. 1849 aus Leipzig.

\section{Urinator arcticus (L.).}

Colymbus arcticus Linn. - Meyer und Helm, Verz. Vög. Kgr. Sachs. p. 123. 
Vom Polartaucher liegen die meisten Daten vor; er ist demzufolge der häufigste unter den drei Seetauchern im Gebiete, das er als seltener Wintergast, hauptsächlich im Jugendkleid, besucht. Alte Vögel sind viel seltener. Von letzteren erhielt J. Fr. Naumann einen aus der Gegend von Leipzig; auch Chr. L. Brehm berichtet, dafs ein solcher (derselbe?) im Febr. 1827 bei Leipzig geschossen worden sei. Ferner bemerkt Reichenbach, dafs ein schönes ausgefärbtes $\nearrow^{r}$ bei Radeburg erlegt wurde, und Held besafs in seiner Sammlung ein \&, am 8. V. 1880 bei Zittau gefangen. In neuerer Zeit wurden nach Schelcher 1 St. im Prachtkleid am 20. V. 1910 auf der Mulde bei Nossen und nach Grimm 1 Ex. im Prachtkleid am 26. V. 1911 auf dem gleichen Flufs bei Tanndorf (bei Colditz) gefangen. Ersterer wurde in den Zoolog. Garten zu Dresden eingeliefert, wo er verendete, der andere gelangte in das Heimatmuseum Leipzig. Hinsichtlich des Herbstzuges sammelte ich folgende Angaben: Reichenbach bemerkt, er komme jung auf die Elbe, was E. J. J. Meyer speziell für die Dresdener Gegend bestätigt. Nach Jacobi [oder Lohse] wurde in den letzten Tagen des Okt. 1888 einer bei Schleufsig (bei Leipzig) erlegt; Jährig erwähnt zwei, die am 15. XI. 1889 bei Räckelwitz (bei Kamenz) beobachtet wurden, von denen einer dem tödlichen Blei erlag. Berge erwähnt nicht weniger als vier: Nov. 1898 wurde 1 Junger bei Ebersbrunn (bei Kirchberg), Nov. 19001 bei Reichenbach i V., Anf. Dez. 19001 mit Jugendspuren im Prachtkleid bei Voigtsgrün (bei Kirchberg) und Nov. 1903 1 Junger bei Falkenstein i. V. erlegt. Rey verzeichnet 2 Leipziger Exemplare vom 6. und 24. XI. 1908, ohne Näheres über deren Alter mitzuteilen, uad Höpfner gedenkt eines jungen Vogels, der 2. XII. 1908 in Geithain (bei Rochlitz) gefangen und dem Leipziger Zoolog. Garten überwiesen wurde, infolge irrtümlicher Bestimmung in diesem Institut unter Eudytes septentrionalis, was Hesse später richtigstellt. Im Zoolog. Museum Dresden stehen zwei Stücke sächsischer Herkunft: 1 mit dem Fundort Moritzburg und $1 \sigma^{x}$, am 14. XI. 1906 auf Staats-Forstrevier Wildenthal bei Eibenstock tot aufgefunden. Am 17. XI. 1914 flog ein Junger in Oederan an, verletzte sich und wurde gefangen.

\section{Urinator lumme (Gunn.).}

Colymbus septentrionalis Linn. - Meyer und Helm, Verz. Vög. Kgr. Sachs. p. 124.

Der Nordseetaucher ist gleichfalls seltener Gast. Noch mehr als beim vorigen tritt die auffällige Erscheinung zutage, dafs fast nur Junge erbeutet werden. Reichenbach scheint ihn öfter erhalten zu haben, denn er versichert, er sei bei Moritzburg, auf der Elbe und an anderen Orten, auch bis Wurzen vorgekommen; ebenso führt ihn E. J. J. Meyer als jung auf der Elbe vorkoinmend auf. Nach Helm wurde am 19. XI. 1888 einer 
ins Zoolog. Museum zu Dresden gebracht, und in der Tat befindet sich hier ein junges \&, „Dresden 1888" datiert. Nach Hennicke wurde dieser Taucher Anfang der siebziger Jahre beim Vorwerk Lindhardt (bei Naunhof), nach Wolff am 22. X. 1892 auf dem Egelsee bei Pirna, nach Berge am 3. XII. 1905 ein Junger bei Wiesenburg (bei Zwickau) und nach Höpfner am 14. XI. 1908 ein Junger in Königsfeld (bei Rochlitz) erbeutet. Schütze sah ein Exemplar aus Kotitz (bei Weifsenberg), und Weifsmantel beobachtete einen am 16. XII. 1909 auf der Mulde bei Rochlitz. Die Tharandter Sammlung enthält ein $\&$ vom 30. XII. 1898 aus Pillnitz (bei Dresden), die Leipziger nach Hesse einen Jungen vom Nov. 1839 aus Leipzig.

\section{Colymbus cristatus L.}

Podiceps cristatus (Linn.). - Meyer und Helm, Verz. Vög. Kgr. Sachs. p. 122.

Dem Haubentaucher gewähren die grofsen Teichblänken des Flachlandstreifens, der den Norden des Gebiets bildet, Unterkunft und Nahrung in nicht geringer Menge. Hier besiedelt er als ziemlich bäufiger Brutvogel alle bedeutenderen Teiche, wie den Horstsee bei Wermsdorf, die Moritzburger Teiche und die der lausitzischen Niederung, die kleineren dagegen seltener und bis zu völligem Ausbleiben unregelmäfsig, soweit seine Ansiedelungsabsichten nicht schon ohnehin mit Pulver und Blei vereitelt werden. Dadurch fehlt er manchem Teichgebiet, das geeignet wäre, ihn zu beherbergen, oder ist doch sehr zurückgegangen. Im Gebirge steigt er als Brutvogel (auf dem Gr. Teich bei Grofshartmannsdorf) bis zu $500 \mathrm{~m}$ Höhe an; auf dem Filzteich fehlt er. Die Ankunft erfolgt ziemlich regelmäfsig Ende März, der Abzug im Oktober, selten später.

\section{Colymbus grisegena Bodd.}

Podiceps rubricollis (Gm.). - Meyer und Helm, Verz. Vög. Kgr. Sachs. p. 123.

Der Rothalstaucher verlangt von seinen Brutteichen einen üppigen Saum höherer Teichvegetation, stellt aber hinsichtlich ihrer Grölse bei weitem nicht so hohe Anforderungen wie voriger, sodafs er im Niederland entschieden häufiger und verbreiteter ist als der Haubentaucher, ins eigentliche Bergland dagegen nicht aufsteigt, weil ihm die Ufer der Bergwässer zu kahl sind. Die höchsten Brutplätze, die ich ermitteln konnte, übersteigen daher $300 \mathrm{~m}$ Höhe nicht; es sind die nach Kramer von ihm regelmäfsig bewohnten Teiche bei Burkersdorf in der Lausitz. Von Mitte bis Ende März an pflegt er zu erscheinen und im Oktober unbemerkt abzuwandern. Als spätestes Vorkommen notierte ich 21. X. 19064 Junge auf dem Hegeteich bei Narsdorf, die daselbst erbrütet waren. 


\section{Colymbus auritus L.}

Podiceps arcticus Boie. - Meyer und Helm, Verz. Vög. Kgr. Sachs. p. 123.

Sehr seltener Gast im Gebiet. Die Verworrenheit, die früher die Nomenklatur der beiden als Ohrentaucher bezeichnnten Steifsfüfse (Colymbus auritus L. [= cornutus Naum., arcticus Naum.] und nigricollis $\mathrm{Br}$. [= auritus Naum.]) beherrschte, macht sich auch in unserer sächsischen Literatur bis in die jüngste Zeit bemerkbar. So berichtet z. B. Etzold in Groitzsch, im Juli 1890 seien 2 bei Frohburg beobachtet worden. Hennicke hielt ihn für einen Brutvogel der Rohrbacher Teiche, weil er ihn einmal beobachtete und ein Junger erlegt wurde. Nach v. Vietinghoff trägt er zur Belebung der Teiche um Neschwitz (Lausitz) bei u. s. w. Alle diese Fälle beziehen sich auf den Schwarzhalstaucher; sie sind nur durch den falsch angewendeten Namen hierher gestellt worden. Zuverlässigere Vorkommen dieses nordischen Tauchers kenne ich nur folgende: Der alte Chr. L. Brehm schreibt, sein Sohn, „der Pharmazeut", habe in den letzten Tagen des September 1845 einen im ersten Herbstkleid bei Limbach geschossen. R. Tobias berichtet $1853^{1}$ ), es habe sich einmal ein Pärchen bei Herrnhut gezeigt. Held besafs in seiner Sammlung ein im Herbst 1875 lebend erhaltenes \& aus Zittau. Nach Jährig fand man Ende der siebziger Jahre einen tot in einem Garten in Kameuz. Bachmann weifs von einem zu berichten, der am 13. I. 1893 auf der Elster bei Plauen angefroren war, und Berge erwähnt 3 Stück, die am 19. IV. 1896 auf einem Teich bei Schönheide (bei Eibenstock) erlegt wurden. Ein Belegexemplar, $\$$ 14. 1. 1888 aus Grumbach (bei Willsdruff), das schon Wolff und Nitsche erwähnen, besitzt die Sammlung der Forstakademie Tharandt.

\section{Colymbus nigricollis (Brehm).}

Podiceps nigricollis Sundev. - Meyer und Helm, Verz. Vög. Kgr. Sachs. p. 123.

Das Vorkommen des Schwarzhalstauchers, der als ziemlich häufiger Brutvogel viele grölsere Teichgebiete besiedelt, wird charakterisiert durch die aufserordentlichen Schwankungen, die sich von Jahr zu Jahr im Bestand der Brutpärchen geltend machen, eine Tatsache, die jedem Beobachter geläufig und von vielen Lokalfaunisten gewürdigt ist. Diese auffällige und schwer erklärbare Erscheinung zeigt sich zwar auch bei anderen Arten, selten aber in dem Umfang wie beim Schwarzhalstaucher. Kramer fand ihn immer in der Nähe von Lachmöwenkolonien und stellte sein Verschwinden nach dem Erlöschen jener fest.

1) Tobias nennt diese Vogel ausdrücklich „Colymbus cornutus Licht.", wăbrend or den Schwarzhals „auritus" nennt. 
Doch findet er sich auch auf Teichen, wo solche Kolonien fehlen, neigt aber selbst dazu, Kolonien zu bilden. Er verlangt reiche Vegetation an den Teichufern und liebt gröfsere Blänken, auf denen er sich im Gegensatz zu $C$. grisegena auch im Sommer gern zeigt. Sein Brüten ist nachgewiesen von Held für die Teiche von Burkersdorf (Lausitz), von Kramer bis 1895 regelmälsig für die bei Grofshennersdorf, von Kramer vereinzelt, von Hantzsch nicht selten für die bei Königswartha, von Helm in gröIserer Anzahl für den Dippelsdorfer Teich bei Moritzburg, von Hesse für Rohrbach, den Müncherteich bei Grimma, jedoch schwankend, von Helm für die Frohburger Teiche und von mir für den Gr. Teich.bei Grofshartmannsdorf, dem mit $500 \mathrm{~m}$ Höhe anscheinend höchsten Brutplatz in Sachsen. Ferner beobachtete ich ihn zur Brutzeit, am 3. VI. 1914, in einigen Exemplaren auf dem Deutschbaselitzer Teich (bei Kamenz) und sowohl 1909 als auch 1910 bis 22. V. auf dem Horstsee bei Wermsdorf. Nach einem nach Hesse im Zool. Institut in Leipzig befindlichen pullus aus Connewitz (bei Leipzig), Juli 1846, hat er früher auch da gebrütet. Die Ankunft erfolgt im April, der Abzug im Oktober. ${ }^{1}$ )

\section{Colymbus nigricans Scop.}

Podiceps minor (Gm.). - Meyer und Helm, Verz. Vög. Kgr. Sachs. p. 123.

Der Zwergtaucher bewohnt die meisten unserer stehenden Gewässer, von den verschilften Winkeln unserer Grofsteiche an bis herab zu kleinen Dorftümpeln, wenn sie nur recht dicht bewachsen sind, und wird dadurch der verbreitetste Taucher. Er fühlt sich in der von Cyperaceen, Simsengewächsen, Schachtelhalmen und verschiedenen Gräsern zusammengesetzten Flora der Gebirgsteiche ebenso wohl wie im Schilf und Rohr des Niederlandes und geht infolgedessen höher hinauf als seine Verwandten. Ich fand ihn im Sommer 1913 als Brutvogel in Grofshartmannsdorf $(500 \mathrm{~m}), 15$. V. 1913 auf dem Filzteich bei Schneeberg, Berge bei Cottenhaide i. Vogtland $(700 \mathrm{~m})$. In der niederen Lagen, z. B. nach Hesse bei Leipzig, nach Neumann und Grünewald bei Grofsenbain, überwintert er, in den höheren wandert er mit Eintritt rauherer Witterung, im Oktober und November, ab und kehrt im Februar oder März zurück.

\section{Stercorarius pomarinus (Tem.).}

Lestris pomarina Temm. - Meyer und Helm, Verz. Vög. Kgr. Sachs. p. 125.

1) Ein von mir Orn. Monatsschr. 1911 p. 445 veroffentlichter Beobachtungstermin ,13. XI.“ beruht auf einem Druck- resp. Schreibfebler; es mufs heifsen: 18. X. 
Die Mittlere Raubmöwe ist für unser Sachsen als seltener Gast zu betrachten. Zweifellos sind in der Literatur öfter Verwechselungen der bei uns nachgewiesenen drei Raubmöwenformen enthalten, sodafs die ohnehin spärlichen literarischen Nachweise kein gutes Bild des Vorkommens zu geben vermögen, vielmehr mit einiger Vorsicht zu benutzen sind. Erbeutet wurde dieser Vogel nach Schach am 6. X. 1851 in einem jungen Exemplar bei Crimmitschau, nach Neumann und Grünewald am 4. IX. 1887 eine Junge vom selben Jahr auf Adelsdorfer Flur (bei Grofsenhain) und nach H. Naumann am 30. XI. 1892 bei Kleinboblitz (bei Bautzen). Jährig besafs in seiner Sammlung eine am 22. IX. 1886 bei Biehla (bei Kamenz) erlegte und sagt, sie sei früher wiederholt an den Teichen bei Kamenz festgestellt worden.

\section{Stercorarius parasiticus (L.).}

Lestris parasitica (Linn.). - Meyer und Helm, Verz. Vög. Kgr. Sachs. p. 125.

Ebenfalls seltener Gast. J. F. Naumann bezeichnet sie in seinem grofsen Werke als in Sachsen und in der Lausitz erlegt, bleibt uns aber leider die Angabe genauerer Fundorte schuldig, sodals sich die Vorkommnisse faunistisch nicht verwerten lassen. Nach Mitteilungen Jährigs wurde diese Raubmöwe ca. 1882 bei Brauna (bei Kamenz) erlegt; auffallenderweise soll sich der Vogel etwa 14 Tage am dortigen Teich gezeigt haben. H. Naumann berichtet vom selben Jahre den Fang eines anderen in nächster Nähe von Bautzen und versichert, am 17. IX. 1893 sei einer bei Grofsseitschen (bei Bautzen) geschossen worden. Hesse erwähnt die Erbeutung eines Stückes von Anf. Okt. 1909 bei Zuckelhausen (bei Leipzig). Nach Schelcher wurde eins am 3. X. 1910 bei Grofsenhain gefangen und in den Zoolog. Garten zu Dresden eingeliefert, und nach Grimm erbeutete man Ende Aug. 1912 eine Junge bei Collm (bei Oschatz). Eine ebensolche, die am 19. IX. 1912 in Falkenau (bei Flöha) tot aufgefunden worden war, untersuchte ich beim Präparator Bär in Oederan.

\section{Stercorarius cepphus Brünn.}

Die Kleine Raubmöwe, wie ihre Verwandten ein seltener herbstlicher Gast, ist erst neuerdings sicher für unser Gebiet nachgewiesen worden, obwohl sie kaum so selten sein dürfte, sondern öfters verkannt worden sein mag. In seiner Liste der sächsischen Vögel (1816) führt schon Mosch eine Möwe „Larus

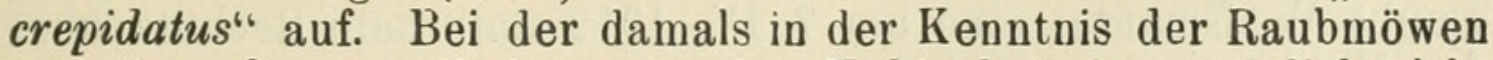
und ihrer Synonymik herrschenden Unklarheit ist natürlich nicht zu ersehen, welche der beiden Formen [parasiticus (L.) und cepphus (Brünn.)] gemeint ist. Für letztere allein wird der Name „crepidatus" erst seit 1822 (Brehm, Beitr. z. Vögelkunde III. p. 861) gebraucht. 
J. F. Naumann bemerkt, sie sei einzeln in Sachsen vorgekommen, ohne das Gebiet näher zu nennen. In der Regel scheint Naumann mit der unklaren Gebietsangabe „Sachsen“ die seinem Vaterland benachbarte preufs. Provinz gleichen Namens zu meinen, weshalb diese seine Angaben hier wertlos sind.

Im Zoolog. Museum Dresden befindet sich ein Stück im ersten Jugendkleid vom 20. IX. 1911 aus Ulberndorf (bei Dippoldiswalde). Sein Vorkommen war von Schelcher bereits irrtümlich unter St. parasiticus veröffentlicht worden. Nachdem inzwischen Jacobi das Tier als cepphus erkannt hatte, berichtigt Schelcher seinen Irrtum und bezweifelt gleichzeitig die Richtigkeit der Bestimmung zweier von ihm gleichfalls als parasiticus bezeichneter Exemplare (juv. 23. IX. 1909 Lausa bei Dresden, juv. Ende Sept. 1909 Meifsen) mit dem Hinweis, dafs es sich hier ebenfalls um Stücke von cepphus zu handeln scheine. Die Artzugehörigkeit steht sonach noch offen.

\section{Larus glaucus Brünn.}

Larus glaucus Brünn. - Meyer und Helm, Verz. Vög. Kgr. Sachs. p. 125.

Im Gebiet sehr seltener Gast. Mosch führt sie 1816 in seinem Verzeichnis der sächsischen Vögel ohne nähere $\mathrm{Be}$ merkungen auf. J. G. Neumann bezeichnet sie als ,höchst selten bei Zittau" vorkommend. Da die Angaben dieses Schriftstellers fast nur auf dem Inhalt seiner und ihm bekannter anderer Sammlungen basieren, scheint er demnach doch ein Exemplar aus der Zittauer Gegend gekannt zu haben. Neuere Nachweise fehlen.

\section{Larus argentatus Brünn.}

Larus argentatus Brünn. - Meyer und Helm, Verz. Vög. Kgr. Sachs. f. 125.

Die Silbermöwe ist seltener Gast. Nach Haake wurden im Frühjahr 18472 Stück bei Eibenstock erlegt. Neumann und Grünewald konstatierten eine am 31. I. 1886 auf dem Strafsenteich bei Kalkreuth (bei Grofsenhain). Eras teilt mit, dafs am 8. XII. 1887 eine bei Höckendorf (bei Dippoldiswalde) erlegt worden sei. Nach Weidemüller wurde im gleichen Jahr eine am Walkteich bei Stollberg (Erzgeb.) geschossen, nachdem die Art schon früher hier erbeutet worden war. M. Naumann beobachtete sie am 17. III. 1888 bei Meifsen elbaufwärts fliegend, und Rey glaubt 2 am 3. IV. 1901 bei Klinga (bei Leipzig) nach NW. ziehende grofse Möwen zu dieser Art rechnen zu können. Ebenso glaubt Weifsmantel eine am 7. IV. 1912 unterhalb Rochlitz über der Mulde gesehen zu haben.

\section{Larus marinus L.}

Mosch erwähnt die Mantelmöwe als „bei Laubegast (bei Dresden) gesehen", verzeichnet sie in seiner Liste jedoch nicht. 
Ich wage nicht, auf diese unsichere Angabe hin die Art als für das Gebiet nachgewiesen zu betrachten.

\section{Larus fuscus $\mathrm{L}$.}

Larus fuscus Linn. - Nachtrag zu Meyer und Helm, Verz. Vög. Kgr. Sachs. [1896] p. 2.

Seltener Gast im Gebiet. Bisher liegen nur Nachrichten vom Vorkommen junger Vögel vor. Nach Baer befindet sich ein junges $\sigma^{x}$ aus Königswartha (Lausitz) in der Sammlung des Barons von Löbenstein in Lohsa. Schwarze, Präparator am Kgl. Zoolog. Museum Dresden, beobachtete 18944 Stück auf der Elbe, von denen er zwei Junge erlegte, die sich im Museum befinden („,Kaditz 1894"; „Uebigau 9. XI. 1894“). Berge nennt folgende Fälle der Erbeutung junger Heringsmöwen im Gebiet: Oktober 1898 bei Burgstädt, Ende Nov. 1900 bei Eichicht (bei Adorf i. V.), Anfang Oktober 1902 bei Zwickau und Oktober 1905 ebenda.

\section{Larus canus L.}

Larus canus Linn. - Meyer und Helm, Verz. Vög. Kgr. Sachs. p. 125.

Die Sturmmöwe ist seltener Gast, doch dürften von allen Seemöwen sie und die Heringsmöwe das Gebiet am ehesten besuchen. Schon Mosch nennt sie in seinem Verzeichnis. Held besafs in seiner Sammlung 1 \& vom 20. I. 1888 aus Kleinschönau (bei Zittau); im Zoolog. Museum Dresden befindet sich 1 junges $\sigma^{7}$ vom 6. II. 1879 aus Loschwitz (bei Dresden). Strohbach versichert, am 9. II. 1888 sei eine bei Glösa (bei Chemnitz) erlegt und nach H. Naumann wurde am 15. XII. 1893 ein altes $\sigma^{7}$ tot bei Löbau gefunden. Hennicke gibt an, am 13. IV. 1890 zwei bei Rohrbach (bei Leipzig) beobachtet zu haben, und Berge berichtet, dafs bei Zwickau je eine im Dezember 1903 und Januar 1904, bei Crossen (bei Zwickau) eine im Nov. 1895 erlegt worden seien.

\section{Larus ridibundus L.}

Xema ridibundum (Linn.), - Meyer und Helm, Verz. Vög. Kgr. Sachs. p. 126.

Die Lachmöwe ist an den Grofsteichen des Niederlandes zur Zugzeit eine ziemlich häufige Erscheinung und während der Brutperiode auch in der weiteren Umgebung der Kolonien nicht selten. Ganz besonders gilt dies neben dem Elblauf für die Teichgegenden der Lausitz, wo sich auf sächsischer Seite gegenwärtig kein Brutplatz befindet, ich aber Anfang Juni allenthalben über den Teichen, Feldfluren, selbst über Holzzchlägen herumstreichende Möwen sah. Vermutlich stammten sie von den nahen Kolonien jenseits der Grenze (Ullersdorf, Klitten, Mönau, Koblenz, Neudorf in der preufsischen Oberlausitz) oder waren nicht zur 
Fortpflanzung geschritten. Über sächsische Brutstätten der Lachmöwe ist mir folgendes bekannt geworden: Eine starke Siedelung befand sich früher nach K. A. Israel auf dem Grofsteich bei Burkersdorf (bei Ostritz) und dem ihm benachbarten Grofsteich von Grofshennersdorf. 1860 und früher war sie sehr stark, von 1875 an erschienen die Vögel vermindert, blieben teilweise auch ganz aus. 1887 wurden die Nester durch Hochwasser zerstört, und die folgenden drei Sommer zeigten sich nur noch 2-6 Paare, bis 1890 die Siedelung erlosch. Eine andere lausitzische Kolonie, 200 Paare umfassend, gründete sich nach Hantzsch 1902 auf dem Mühlenteich bei Commerau (bei Königswartha), doch scheint sie nicht lange bestanden zu haben, denn 1914 fand ich dort keine vor, ebensowenig konnten sich Commerauer Einwohner des Möwenbrutplatzes erinnern. Eine alte Brutstätte ist die des Spitalteiches bei Adelsdorf (bei Grofsenhain), die 1878 nach M. Neumann ca. 1000 Paare umfafste, in welchem Jahre am 21. IV. 243 , am 6. V. gegen 400 Eier gesammelt wurden. Von ihr splitterten Teile $a b$, welche die südöstl. gelegenen Teiche zwischen Kalkreuth und Schönfeld besiedelten. Gegenwärtig sind, wie mir Jacobi 1913 mitteilt, beide Kolonien besetzt, erstere mit ca. 50 Paar. Ein weiterer Brutplatz befindet sich auf den Moritzburger Teichen. Der Dippelsdorfer Teich wird nach Helm als Nistplatz bevorzugt; zuweilen brüten auch Möwen auf dem Frauenteich bei Bärwalde. 1840 bestand die Kolonie, wie Chr. L. Brehm von Dehne erfuhr, aus 500 Paaren und 1893 schätzte Helm im grofsen und ganzen dasselbe. Die Mitglieder dieser Kolonie hauptsächlich besuchen den Elbstrom bis hinauf in die Sächsische Schweiz und gaben verschiedenen Beobachtern Veranlassung zu der irrigen Ansicht, die Möwen brüteten auf den Elbhegern. Nach Hülsmann brüteten 1878 etwa 30-40 Paare im Burkartshainer Teich (bei Wurzen) ohne wiederzukommen und zwar auf einer Sandbank. Er schreibt damit aber der Lachmöwe fälschlich die Nistweise der eigentlichen Seemöwen zu. Gleichfalls erloschen ist die Siedelung auf den Rohrbacher Teichen (bei Belgershain), über die uns Hennicke berichtet. Er beobachtete 1889 über $30 \mathrm{St}$. bis Ende Juli, vermochte aber das Brüten nicht nachzuweisen, 1890 erschienen viele Hunderte und es wurden zahlreiche Nester gefunden, in den nächsten zwei Jahren nahm ihre Zahl rasch ab, dann kamen sie nicht wieder. Schliefslich wäre noch der Kolonie auf den Frohburger Teichen zu gedenken, die von verschiedenen Schriftstellern erwähnt wird. Sie bestand bis 1913, dann siedelten die Mitglieder teilweise nach den Haselbacher Teichen (in Sachs.Altenburg) über, wo sie gegenwärtig auch nicht mehr brüten. Endlich brüteten in neuester Zeit Lachmöwen auf dem Gr. Teich bei Grofshartmannsdorf und zwar $19124,19132,1914$ ebenfalls 2 Paare. Ferner behauptete Rey ihr Brüten für die Teiche bei Grethen (bei Grimma), was Hesse für 1907 und 1908 jedoch nicht bestätigt fand. Es bestehen also gegenwärtig im Königreich 
4 Kolonien: Adelsdorf, Kalkreuth, Moritzburg und Grofshartmannsdorf. Von den Brutplätzen verschwinden sie gewöhnlich Ende Juli; der eigentliche Zug setzt aber erst später ein und dauert bis Oktober, selten länger. Im März erfolgt die Ankunft, doch erhielt Rörig schon 2 am 6. II. im Königreich Sachsen geschossene \&.

\section{Rissa tridactyla (L.).}

Rissa tridactyla (Linn.). - Verz. Vög. Kgr. Sachs. p. 126. Die Dreizehenmöwe besucht, meist wohl durch widrige Windströmungen verschlagen, das Gebiet als seltener Gast. Mosch führt sie in seinem Verzeichnis der sächsischen Vögel ohne Bemerkungen auf. Neumann und Grünewald erwähnen ihr Vorkommen vom 7. XI. 1887 auf dem Kl. Spitalteich bei Grofsenhain, nach Päfsler wurde am 15. IV. 1889 eine bei Breitenbach (bei Meerane) auf einer Wiese tot aufgefunden, nach Berge erlegte man sie in je einem Exempl. im Jan. 1896 bei Cainsdorf (bei Zwickau) und im Febr. 1900 bei Werdau, und Höpfner untersuchte eine Junge, die am 5. XI. 1901 tot bei Penna (bei Rochlitz) gefunden war. Im Zoolog. Museum Dresden befindet sich ein Exempl. vom Febr. 1868 aus Dresden, und nach Hesse enthält die Sammlung des Zoolog. Instituts Leipzig eine Junge vom Nov. 1845 aus Lützschena (bei Leipzig).

\section{Gelochelidon nilotica (Hasselqu.).}

Sterna anglica Mont. - Nachtrag [1896] zu Meyer und Helm, Verz. Vög. Kgr. Sachs. p. 2.

Für das Gebiet liegt nur eine Notiz vom Auftreten dieser Art vor: Der Berichterstatter für Meyer und Helms Jahresberichte H. Naumann in Bautzen bezeichnet die Lachseeschwalbe als am 10. VIII. 1891 bei Königswartha, am 13. VIII. 1891 bei Klix (Lausitz) vorgekommen und nennt ein Jahr darauf den ersteren Ort als Fundstelle der Art wieder. Den sehr dürftigen Nachrichten fehlt jede Bemerkung, aus der hervorgehen könnte, ob Vögel erbeutet wurden oder ob es sich um Beobachtungen oder gar nur Naumann zugetragene Mitteilungen handelt. Meyer und Helm nehmen zwar ihre Richtigkeit an, weil sie auf Grund der Angaben die Seeschwalbe in ihr Verzeichnis der sächsischen Vögel aufnehmen, doch beweist dies noch nicht, dafs sie dieselben nachgeprüft haben. Ohne das etwaige Vorkommen der Art im Gebiet als ausgeschlossen bezeichnen zu wollen, bietet mir doch die Fassung jener Notizen nicht die notwendige Sicherheit, um die Art den sächsischen Vögelu einreihen zu können.

\section{Sterna cantiaca Gm.}

In seinem Verzeichnis führt Mosch auch "Sterna canescens" auf und nennt sie an anderer Stelle unter den bei Reinhardtsdorf beobachteten Vögeln. Bei der schon erwähnten Unzuverlässigkeit Moschs sehe ich jedoch hierin keinen vollgültigen Nachweis. 


\section{Sterna hirundo L.}

Sterna fluviatilis Naum. - Meyer und Helm, Verz. Vög. Kgr. Sachs. p. 126.

Die Flufs-Seeschwalbe brütete bis in die neuere Zeit auf den Kiesbänken und Hegern der Elbe und unteren Mulde, vereinzelt auch da und dort an gröfseren Teichen. Doch ist mir für die Gegenwart kein regelmälsiger Brutplatz bekannt. Ende Mai 1909 und Juni 1910 bin ich ihretwegen mit dem Dampfer elbaufwärts bis Wehlen, Juni 1914 abwärts bis Riesa gefahren ohne auch nur eine zu sehen. Die Zeiten, in denen A. B. Meyer sagen konnte, sie sei im Sommer ,sehr zahlreich zwischen Dresden und Blasewitz", wo Jugl sie häufig zwischen Dresden und Niederwartha fand, Wünsche ihr Brüten auf einer Insel bei Pirna feststellte und Hiller sie als ,in grofsen Massen“ auf der Insel bei Pillnitz nistend bezeichnet, sind durch die Ausbaggerungen und Uferregulierungen unwiederbringlich dahin. Auch von der unteren Mulde, wo sie nach Heym bei Wurzen, nach Hülsmann bei Nischwitz brütete, melden neuere Beobachter nichts mehr, obschon sie hier ungestörter ist als auf der Elbe. Auf den Teichen scheint sie sich, da hier Kiesbänke fehlen, gern an Lachmöwenkolonien anzuschliefsen und sich deren Nistweise zu eigen zu machen, also auf Stubben oder angetriebenem Pflanzenwust zu brüten. Ende der achtziger Jahre brüteten einige Paare nach Hiller auf dem Dippelsdorfer Teich bei Moritzburg, woher das Zool. Museum Dresden 2 Eier besitzt, Hennicke fand sie als häufigen Brutvogel auf den Rohrbacher Teichen während der Anwesenheit der Möwensiedelung, und Hantzsch konstatierte einzelne Brutpaare unbeständig bei Caminau (bei Königswartha). Während des Zuges erscheint sie gelegentlich an allen gröfseren Gewässern, zuweilen auch ziemlich häufig, doch nie so regelmäfsig wie ihre dunkle Schwester. Sie kommt im Mai und wandert vom Juli an wieder $a b$, wobei sich einzelne Exemplare bis in den September hinein verweilen; Hesse sah sogar 25.--27. XII. 1906 eine bei Möckern (bei Leipzig).

\section{Sterna minuta L.}

Sterna minuta Linn. - Meyer und Helm, Verz. Vög. Kgr. Sachs. p. 126.

J. F. Naumann charakterisierte ihr ehemaliges Vorkommen in Sachsen sehr treffend, indem er sagte, sie bewohne die Elbe bis über Dresden hinauf und die Mulde stellenweise bis in die Gegend von Wurzen. Sie teilte das Schicksal der Flufs-Seeschwalbe, mit der sie auch die Nistplätze gemeinsam, aber in geringerer Zahl besiedelte, und kommt gegenwärtig regelmäfsig nirgends mehr vor. In den achtziger Jahren fanden sie an der Elbe Neumann und Grünewald bei Seufslitz, A. B. Meyer zahlreich zwischen Dresden und Blasewitz, Fickert in ziemlicher Menge jeden Sommer auf der Pillnitzer Insel brütend, an der Mulde Heym 
als regelmäfsigen Brutvogel bei Wurzen, Hülsmann auf einer Insel bei Nischwitz zwar abnehmend, aber 1888 noch immer in 3 Paaren. Nach Hiller brüteten sogar 2 Paare auf dem Dippelsdorfer Teich (bei Moritzburg). Wiglesworth vermutete ihr Brüten auf der Pillnitzer Elbinsel und fand am 17. VII. 1892 auf der Insel bei Gauernitz (bei Dresden) ein ca. 2 Tage altes Junge und $1 \mathrm{Ei}$. Wie mir Voigt mitteilt, fand er die ZwergSeeschwalbe 1908 noch auf Schotterbänken nördlich von Wurzen, woher stud. Pönitz auch ein Gelege dieser Art besitzt, später aber nicht mehr. Über Ankunft und Abzug schweigt sich die Literatur völlig aus, nur einige auffällig späte Herbstzugdaten sind mir bekannt geworden: G. Thienemann sen. beobachtete noch im September eine bei Dresden, nach Bauer wurden am 10. IX. 18882 bei Friesen (bei Reichenbach) beobachtet und eine davon geschossen, und Berge notiert eine rom September 1902 aus Zwickau.

\section{Hydrochelidon leucoptera (Schinz).}

Hydrochelidon leucoptera M. u. Sch. - Meyer und Helm, Verz. Vög. Kgr. Sachs. p. 126.

Sehr seltener Gast. Päfsler versichert, am 2. VI. 1887 sei eine Weifsflügelige Seeschwalbe bei Breitenbach (bei Meerane) in einem Pfahleisen gefangen worden. Ich halte Päfsler für vogelkundig genug, um eine Verwechselung als ausgeschlossen zu betrachten Ferner behauptet Zimmermann, am 12. V. 1912 2, am 16. V. 1912 nochmals eine an den Frohburger Teichen beobachtet zu haben.

\section{Hydrochelidon nigra (L.).}

Hydrochelidon nigra Boie. - Meyer und Helm, Verz. Vög. Kgr. Sachs. p. 127.

Die Trauer-Seeschwalbe ist als Brutvogel eine bezeichnende Erscheinung der stark bewachsenen, nicht zu kleinen Teiche, ist daher mehr an die Gewässer des Lausitzer Tieflandes gebunden und fehlt den gepflegten Karpfenteichen des Westens (Frohburg, Rohrbach, Wermsdorf, Moritzburg u. s. f.) und den Flüssen. Nach Baer konstatierte sie Kramer als häufigen Brutvogel an den Teichen des Lausitzer Tieflandes, Lübeck nennt sie einen ziemlich häufigen Brutvogel der Teiche bei Brauna, Grüngräbchen und Schwepnitz ${ }^{1}$ ), nach Fritzsche brütete sie 1877 in ziemlicher Zahl bei Böhla (bei Ortrand), Hantzsch fand kleine Kolonien im

1) Diese Brutstătten sind auf den Karten, die Krobn seiner Abhandlung über die Verbreitung der Mrwen und Seeschwalben in Deutschland beifügt (Orn. Monatsschr. 1905 p. 206 ff.), unrichtig eingezeichnet (Vergl. l. c. 1909 p. 283), ebenso die Mőwenbrutstátten Breitenbach und Burkersdorf. 
Grenzteich bei Königswartha und bei Holscha, ich selbst ebensolche im Juni 1914 bei Caminau (bei Königswartha) und bei Quoos. Hennicke beobachtete während der Jahre, in denen Lachmöwen in Rohrbach brüteten, alljährlich im Sommer 12 bis 14 Paare daselbst und vermutete deshalb ihr Brüten. Zur Zugzeit erscheint sie in allen gröfseren Teichgebieten regelmäfsig, kommt von Ende April an bis Ende Mai, nicht selten einzeln auch in Juni, daselbst vor und verschwindet mit Ende August oder im September. Im Zool. Museum in Dresden befindet sich sogar eine Junge vom 3. X. 1899 aus Moritzburg.

\section{Phalacrocorax carbo (L.).}

Carbo cormoranus M. u. W. - Meyer und Helm, Verz. Vög. Kgr. Sachsen p. 124.

Der Kormoran konnte auch für frühere Zeiten nicht als Brutvogel des Königreichs Sachsen nachgewiesen werden, sondern besuchte dasselbe nur als seltener Gast. Nach Reichenbach wurde er bisweilen auf dem Grofsen Teiche bei Moritzburg (bei Dresden) und auch bei Leipzig erlegt. Demzufolge führt ihn auch E. J. J. Meyer für Dresden auf. Schach berichtet, am 3. I. 1853 seien aus einem Trupp von 7 Stück, der sich bereits vier Wochen auf der Mulde bei Waldenburg aufgehalten habe, ein $\&$ erlegt und ein $\sigma^{\top}$ geflügelt worden. Dehne kannte diese Tatsache ebenfalls und fügt hinzu, dafs auch am 31. X. 1853 ein sehr altes $q$ bei „Kostebaude“ (soll heifsen Cossebaude bei Dresden) unfern der Elbe lebend von einem Baum geschüttelt worden sei. Später führt ihn Heym ziemlich nichtssagend als "seltenen Durchzügler" bei Wurzen auf, und Berge versichert, am 7.X. 1899 sei vom Waldwärter in Neudorf (bei Oberwiesenthal) einer aus einem Flug von vier geschossen worden. Nach Hesse befindet sich im Zoolog. Institut Leipzig ein Junger vom 18. XI. 1862 aus Lützschena (bei Leipzig). Die Tharandter Sammlung bewahrt ein Stück vom Herbst 1869 aus dem Crostauer Revier (bei Schirgiswalde) auf.

\section{Sula bassana (L.).}

Sula bassana (Linn.) - Meyer und Helm, Verz. Vög. Kgr. Sachs. p. 124.

Zweimal im Gebiet festgestellter sehr seltener Gast. J. G. Neumann erwähnt den Bafstölpel als bei Zittau vorgekommen, und nach Reichenbach wurde einer Anfang Dezember 1824 bei Knauthain (bei Leipzig) auf einem Baum gefangen.

\section{Mergus merganser L.}

Mergus merganser Linn. - Meyer und Helm, Verz. Vög. Kg. Sachs. p. 122. 
Der Gänsesäger besucht von November bis März unsere Flüsse, besonders Elbe und Mulde, von allen drei Mergusarten am regelmälsigsten und zahlreichsten. Helm beobachtete beispielsweise im Jan. 1891 auf der Elbe im Weichbilde der Stadt Dresden bis 70 Stück, darunter 25 o $^{x}$ im Prachtkleide, und nach Voigt konstatieren auch er und seine ornithologischen Freunde alljährlich ein Dutzend und mehr auf der Mulde unterbalb Wurzen, überwiegend ơ.

\section{Meryus serrator L.}

Mergus serrator Linn. - Meyer und Helm, Verz. Vög. Kgr. Sachs. p. 122.

Weit seltener als voriger ist der Mittlere Säger; auch er erscheint hauptsächlich auf den genannten Flüssen, doch immer einzeln und unregelmälsig, sodafs er als seltener Wintergast betrachtet werden kann. Reichenbach erhielt allerdings nicht selten Junge, alte $\sigma^{x}$ dagegen selten. Ein schönes $\sigma^{x}$ sandte ihm der Förster Sachse 17. IV. 1835 aus der Gegend von Freiberg, welches sich mit der Fundortsangabe „Mönchenfrei“" (bei Freiberg) heute noch in der Dresdener Sammlung befindet. Aufser diesem wird hier noch 1 우 vom 1. III. 1909 aus Kaditz (bei Dresden) aufbewahrt. Wie Hülsmann berichtet, wurde 1. XI. 18871 Q bei Wurzen erlegt; Marx sen. erhielt ein am 26. VIII. (1) 1888 auf der Elbe erlegtes Exemplar, auch im September 1888 wurde bei Mautitz (bei Riesa) 1 ơ $^{\top}$ geschossen. Peschel glaubte sogar annehmen zu können, dafs sich ein Paar den Sommer hindurch auf der Elbe aufgehalten habe. Nach Jährig schofs man 15. XI. 1889 1 O bei Nebelschütz (bei Kamenz). Berge erwähnt ihn als wiederholt bei Zwickau erlegt.

\section{Mergus albellus $\mathrm{L}$.}

Mergus albellus Linn. - Meyer und Helm, Verz. Vög. Kgr. Sachs. p. 122.

Der Zwergsäger ist, als seltener Wintergast auftretend, für die Zeit vor Neujahr nur einmal nachgewiesen, indem Held in seiner Sammlung $\sigma^{7}$ und $\bigcirc$ vom Herbst 1875 aus Kleinschönau (bei Zittau) besafs, dagegen im Januar regelmälsiger angetroffen worden. So schofs man nach v. Minckwitz 1 junges $\sigma^{x}$ am 15. I. 1887 bei Bennewitz (bei Wurzen), nach Strohbach 1 Stück am 21. I. 1891 bei Chemnitz, nach Hülsmann $1 \sigma^{x}$ am 5. I. 1888 bei . Nischwitz (bei Wurzen), Höpfner beobachtete 1 or vom 18.-20. I. 1893 bei Grimma, Helm ein solches vom 21.-25. I. 1891 auf der Elbe in Dresden, um welche Zeit (26. I. 1891) auch der Oberförster Täger 1 junges $ᄋ$ bei Pillnitz (bei Dresden) schofs, das jetzt in der Tharandter Sammlung steht. Ferner enthält die Sammlung des Zool. Instituts in Leipzig nach Hesse 1 junges 우 vom 16. II. 1870 aus Schleufsig (bei Leipzig). Kramer beobachtete 
je $1 \sigma^{x}$ am 6. und 8. IV. 1896 bei Königswartha und am 20. III. 1898 bei Burkersdorf (bei Ostritz). Wichtrich bemerkte ihn fast alljährlich im Januar an der Mulde unterhalb Wurzen.

\section{Somateria mollissima (L.).}

Somateria mollissima (Linn.). - Meyer und Helm, Verz. Vög. Kgr. Sachs. p. 122.

Die Eiderente ist seltener Gast. Reichenbach teilt mit, dafs sie sich schon bis ins [sächsische?] Vogtland verflogen habe, Oberländer in Greiz habe ein solches Stück ${ }^{1}$ ) in seiner Sammlung. Strohbach sandte dem Zool. Museum Dresden 1 am 13. I. 1889 bei Auerswalde (bei Chemnitz) erlegtes \& und bemerkt, ein ebenfalıs im Januar geschossenes \& der Sammlung Schulze [in Chemnitz?] stamme aus Gablenz (bei Chemnitz). Nach Berge wurde im Oktober 1894 eine solche Ente auf einem Waldteich bei Schönfels (bei Neumark) erbeutet und nach Helm eine Dez. 1908 in Chemnitz völlig ermattet ergriffen und der Städtischen Sammlung überwiesen.

\section{Oidemia fusca (L.).}

Oidemia fusca (Linn.). - Meyer und Helm, Verz. Vög. Kgr. Sachs. p. 121.

Die Samtente ist seltener Durchzügler und Wintergast. Schach beobachtete am 20.X.1850 2 bei Rufsdorf (bei Crimmitschau) und erlegte eine davon. Wünsche besals in seiner Sammlung 1 ㅇ, zu Aufang der siebziger Jahre an der Elbe erlegt, Held in der seinigen $1 \sigma^{x}$, am 24. XI. 1885 bei Zittau geschossen. Auch in der Tharandter Sammlung steht 1 junges $\sigma^{\top}$ vom 7. XI. 1888 vom Tharandter Schlofsteich. Nach Oberförster v. Zebmen schofs man eine Junge am 22. X. 1888 auf dem Heidemühlen-Teich bei Wendischcarsdorf (bei Dippoldiswalde), H. Naumann erhielt 1 am 23. III. 1893 bei Soritsch (bei Bautzen) erbeutetes 우 und Berge bucht eine am 29. XII. 1895 bei Schindmaas (bei Glauchau) erlegte. Beobachtet wurde diese Ente von Hesse am 22. I. 1901 in einem O im Rosental bei Leipzig, am 11. XII., 18. XII. 1905 und 15. I. 1906 [wohl stets dasselbe] in einem ơ bei Rohrbach (bei Belgershain), von Weifsmantel am 16. XI. 1908 in einem Exemplar auf der Mulde bei Rochlitz und angeblich auch von dem allerorts bestätigungsbedürftigen Etzold Anfang April 1889 in der Schnauderaue bei Groitzsch. Nach Voigt beobachteten auch Mitglieder des Leipziger Ornith. Vereins wiederholt einzelne $\sigma^{x}$ auf der Mulde unterhalb Wurzen. Nach einer brieflichen Mitteilung

1) Nach einem Brief Oberlanders an E. F. von Homeyer war es ein adultes $\sigma^{\top}$ (Vergl. E. F. v. Homeyer, Ornithologische Briefe, 1881 p. 245). 
Ein Beitrag zur Kenntnis der Vogelwelt des Konigreichs Sachsen. 215

Jacobis gelangte ein am 17. X. 1914 bei Pommritz erlegtes $\sigma^{\top}$ ad. in vollständigem Prachtkleid in das Dresdener Museum.

\section{Oidemia nigra (L.)}

Oidemia nigra (Linn.). - Meyer und Helm, Verz. Vög. Kgr. Sachs. p. 121.

Die Trauerente berührt das Gebiet auf dem Durchzug als sehr seltener winterlicher Gast. Trotz verschiedener Nachrichten, die das Vorkommen dieser nordischen Ente melden, kenne ich nur einen vollgültigen Nachweis. Nach Höpfner erhielt die Sammlung des Lehrerseminars Rochlitz 1 junges \&, am 17. XI. 1908 bei Rochlitz auf der Mulde erlegt. Nach den mir von Professor Höpfner freundlichst mitgeteilten Kennzeichen des Vogels kann ich die Diagnose nur bestätigen. Aufserdem nennt schon Berge die Art als in $\sigma^{T}$ und $\&$ im Frühjahr 1873 bei Irfersgrün (bei Lengenfeld i. V.) geschossen, kennt jedoch die Vögel nicht aus eigener Anschauung, sondern stützt sich auf das Zeugnis des Ratsförsters Schier. Mosch führt sie unter den bei Reinhardtsdorf (a. d. Elbe) zu beobachtenden Spezies auf, verwechselt sie jedoch möglicherweise mit einer anderen Art. In der Tharandter Sammlung befindet sich 1 \&, im Februar 1881 auf dem Leipziger Markt gekauft, von vielleicht sächsischer Provenienz. ${ }^{1}$ )

\section{Cosmonetta histrionica (L.).}

Mosch führt die Kragenente als bei Reinhardtsdorf (a. d. Elbe) vorkommend auf. Ich erblicke darin keinen zuverlässigen Nachweis.

\section{Nyroca marila (L.).}

Fuligula marila (Linn.). - Meyer und Helm, Verz. Vög. Kgr. Sachs. p. 121.

Die Bergente mufs für das Königreich als seltener Durchzugs- und Wintervogel bezeichnet werden, wenngleich sie etwas häufiger auftritt als etwa Oidemia fusca. Vorwiegend handelt es sich um junge Vögel. Als Zeit ihres Auftretens sind Dezember bis März zu betrachten, doch kommen zuweilen Regelwidrigkeiten vor. So beobachtete Höpfner am 12. IV. 1892 12-14 Stück auf dem Mühlteich bei Altenhain (bei Grimma).

\section{Nyroca fuligula (L.).}

Fuligula cristata (Leach.). - Meyer und Helm, Verz. Vög. Kgr. Sachs. p. 121.

1) Nach Pafsler ist am 3. XI. 18891 Stück bei Haselbach (bei Regis) erlegt. Die bedeutenderen Gewăsser des Haselbacher Teichgebietes liegen jedoch schon jenseits der Grenze im Altenburgischen, mit ihnen wohl auch der Fundort. 
Im ganzen Gebiet, soweit entsprechende Gewässer vorhanden sind, als ziemlich seltener Zugvogel, für die bedeutenderen Flufsläufe auch als gelegentlicher Wintergast bekannt, konnte die Reiherente nur für ein Gebiet als Brutvogel sicher nachgewiesen werden. Dieser Ort sind die Moritzburger Teiche. Hier beobachteten sie Helm und Lionel Wiglesworth - beide damals Assistenten am Zool. Museum Dresden - in den Jahren 1891 und 1892 den ganzen Sommer bindurch. Helm sah Ende Juni 1891 ein Entenweibchen mit Jungen, das er bestimmt für fuligula ansprach, und Wiglesworth stellte am 25. VI. 1892 die Anwesenheit mehrerer $\&$ mit Jungenten auf dem Dippelsdorfer Teiche fest. Auch für die Frohburger Teiche vermutete Helm das Brüten der Reiherente, weil er noch im Juni 1894 solche daselbst antraf, vermochte es jedoch nicht bestimmt nachzuweisen. Ich selbst sah auf diesen von mir viel besuchten Teichen nur einmal die Reiberente zur Brutzeit, nämlich mit Höpfner und Weifsmantel am 9. VI. $19082 \sigma^{x}, 1$ o und am gleichen Tage $1 \sigma^{x}$ unter Tafelenten auf einem der nordwestlichen, jenseits der Grenze im Altenburgischen gelegenen Teiche bei Haselbach. $\mathrm{Zu}$ ungewöhnlicher Zeit, am 5. VIII. 1892 fand nach Baer auch Kramer 12 Vögel dieser Art, darunter 4 alte $\sigma^{7}$, bei Königswartha (Lausitz), und Berge erwähnt 2 Stück, die nach Angabe des Forstassessor Schulze in Hartmannsdorf Aufang August auf dem Filzteich bei Schneeberg erlegt worden sind. In beiden Fällen ist ein Brüten nicht ausgeschlossen. Während die eigentliche Zugzeit auf die Monate März, April und November fällt, begegnet man ihr nicht selten noch im Mai; auch sind zahlreiche Fälle ihresVorkommens während Dezember, Januar und Februar verbürgt.

\section{Nyroca ferina (L.).}

Fuligula ferina (Linn.). - Meyer und Helm, Verz. Vög. Kgr. Sachs. p. 120.

Brandente. Die Tafelente ist neben dem Haubentaucher die erste Charaktergestalt der grofsen, blänkenreichen Teiche und fehlt ihnen nur, wenn es völlig an höherem Uferpflanzenwuchs mangelt, wie z. B. auf dem Filzteich bei Schneeberg. Sie ist zugleich aber auch nach der Stockente die häufigste Brutente. Als höchster Brutplatz dürfte der Gr. Teich bei Grofshartmannsdorf $(500 \mathrm{~m}) \mathrm{zu}$ betrachten sein. Ihre Ankunft ist im Lauf des März zu erwarten, die letzten pflegen mit Ende Oktober oder im November abzuwandern, einzelne überwintern.

\section{Nyroca rufina (Pall.).}

Fuligula rufina Pall. - Meyer und Helm, Verz. Vög. Kgr. Sachs. p. 120. 
Ein Belegstück der als sehr seltener Gast auftretenden Kolbenente besitzt nach Hesse die Sammlung des Zool. Instituts zu Leipzig; es ist ein 우 aus Altenhain ${ }^{1}$ ) (bei Trebsen) von 1845.

\section{Nyroca nyroca (Güld.).}

Fuligula nyroca (Güldenst.). - Meyer und Helm, Verz. Vög. Kgr. Sachs. p. 120.

Als Brutvogel ist die Moorente im allgemeinen eine Eigenheit des sächsischen Ostens, obwohl sie auch im Westen nicht völlig fehlt. Immerhin ist sie hier eine seltene Erscheinung, während sie die Teiche des lausitzischen Niederlandes sowohl regelmäfsig als auch häufiger aufweisen. Man kennt sie als Brutvogel der Teiche von Königswartha nach Kramer und Hantzsch; letzterer nennt besonders die Teiche zwischen den Entenschänkhäusern und Commerau. Ich selbst beobachtete sie nicht selten am 4. VI. 1914 ebenda, einzelner bei Holscha, Radibor und Deutschbaselitz. In der Sammlung der Forstakademie Tharandt befindet sich ein pullus vom 8. VIII. 1878 aus Moritzburg, von wo sie Helm später nicht mehr nennt. Auch auf den Teichen zwischen Frohburg und Eschefeld, wo sie seinerzeit nach Liebe J. Kratzsch so häufig fand, dafs er sie geradezu als die häufigste der brütenden Enten bezeichnen konnte, scheint sie gegenwärtig zu fehlen, da sie weder von Wichtrich noch von Helm als Brutvogel erwähnt wird, auch von mir nicht festgestellt werden konnte. Doch brütet sie auf den benachbarten Haselbacher Teichen jenseits der Grenze. Nach Hülsmann brütete in den achtziger Jahren 1 Paar mehrere Jahre auf einem Teich bei Püchau (bei Wurzen), und Hesse stellte das Brüten eines Paares in den Jahren 1906-1909 auf den Rohrbacher Teichen bei Belgershain fest. Sie erscheint von Ende März an und verschwindet, wie die fast fehlenden Herbstzugdaten vermuten lassen, ziemlich unbemerkt, im Oktober; Hesse beobachtete sie 1906 sogar bis 10. XII.

\section{Nyroca clangula (L.).}

Clangula glaucion (Linn.). - Meyer und Helm, Verz. Vög. Kgr. Sachs. p. 121.

Die Niederung der sächsischen Oberlausitz beherbergt die südlichsten Ausläufer des märkisch-lausitzischen Verbreitungsgebietes der Schellente. Sie ist bis jetzt als Brutvogel nachgewiesen für die Teichgebiete von Klix, Milkel, Königswartha, Milstrich, Deutschbaselitz und Schwepnitz. Für Klix erwähnt sie als seltenen Brutvogel Schuster; bei Milkel beobachtete Kramer 1895 Junge; derselbe konstatierte sie als häufigen

1) In der Originalnotiz (Journ. f. Orn. 1908 p. 265) sagt Hesse, der Fundert liege $1 \frac{1}{2}$ Meilen westlich seines damaligen Beobachtungsgebietes, doch teilt er mir brieflich mit, dafs es $\delta \mathrm{stlich}$ heifsen mulsse. 
Brutvogel bei Königswartha, wo er 22. VII. 18936 noch nicht flugbare Junge fand; Hantzsch stellte sie ebenda vereinzelt fest und bemerkt ihr ständiges Brüten in einem alten Kiefernbestand bei Truppen. Jährig bekam 25. VI. 18882 Junge aus Milstrich (bei Kamenz), wo 4 auf einer Wiese gefangen worden waren, und sandte einen pullus an das Zool. Museum Dresden ein. Bei Deutschbaselitz fand sie nach Baer Kramer als Brutvogel; hier habe ich sie zur Brutzeit, am 3. VI. 1914, auf dem Gr. Teich

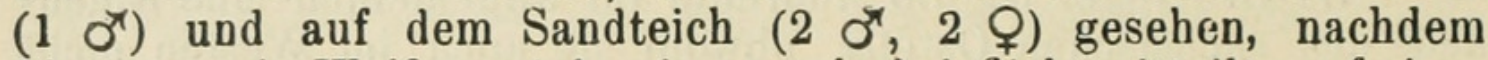
hier vor mir Weifsmantel, wie er mir brieflich mitteilt, auf einer Maiexkursion 16 Pärchen beobachtete und ein Gelege in einer alten Eiche fand. Von Schwepnitz kenne ich sie durch eine briefliche Mitteilung des Waldwärters Ziegler, der mir versichert, dafs man beim Schlagen einer alten Kiefer auf Schwepnitzer Staatsforstrevier in einer Schwarzspechthöhle Enteneier gefunden habe. Auf dem Durchzug zeigt sie sich im März, April, manchmal schon Ende Februar, und im Oktober, November, gelegentlich auch überwinternd.

\section{Nyroca hyemalis (L.).}

Harelda glacialis Leach. - Meyer und Helm, Verz. Vög. Kgr. Sachs. p. 121.

Die Eisente ist sehr seltener Wintergast und wurde in folgenden Fällen für das Gebiet nachgewiesen: In der Sammlung der Forstakademie Tharandt steht ein altes $\sigma^{*}$, in den 40 er Jahren vom Forstinspektor Cotta auf dem Tharandter Schlofsteich erlegt. E. J. J. Meyer nennt sie 1840 unter den Dresdener Vögeln. Mitglieder des Ornith. Vereins in Grofsenhain beobachteten 28. II. 18792 auf den Röderwiesen bei Grofsenhain. Nach K. Krezschmar [oder Held] wurde 1 \% im Dez. 1887 auf der Neifse bei Zittau geschossen. Endlich beobachtete Hesse ein vermutlich jüngeres $\&$ am 11. XII. 1905 bei Rohrbach.

\section{Spatula clypeata (L.).}

Spatula clypeata (Linn.). - Meyer und Helm, Verz. Vög. Kgr. Sachs. p. 119.

Die Löffelente wählt mit Vorliebe grofse Teichlandschaften zur Brutstätte, deren Gewässer aufser reichen Schilfbeständen flache, sumpfige, möglichst in Wiesen auslaufende Ufer aufweisen. Doch tut sie dies mit einer gewissen Regellosigkeit, indem sie auch manchem Teichgebiet fehlt, das diese Merkmale hat. Held berichtet, dafs sie anfangs der siebziger Jahre und 1880 auf den Burkersdorfer Teichen (bei Ostritz) nistete und nennt sie als Brutvogel der benachbarten Teiche bei Grofshennersdorf, wo sie als solchen Kramer in der Zeit nach 1897 nicht mehr fand. Schuster erwähnt sie für Klix, H. Naumann für Milkel und Königswartha als Brutvogel, an welch letzterem Platz sie auch 
Hantzsch und ich nicht selten fanden. Nach Helms und eigenen Notizen ist sie bei Frohburg als Brutvogel zwar garnicht selten, jedoch unregelmäfsig; Weifsmantel fand hier 30. V. 19122 Gelege am Ziegelteich. Nach Baer erhielt sie Präparator Aulich 11. V. aus Baruth, Hülsmann erlegte von 3 ein junges 우 im Juni 1885 bei Wurzen; ich selbst beobachtete sie Sommer 1908 hindurch auf dem Horstsee bei Wermsdorf, 1909 und 1910 ebenfalls bis in den Mai hinein, Juni 1912 und 1914 auf den Grofshartmannsdorfer Teichen, 3. VI. 1914 auch auf dem Grofsteich bei Deutschbaselitz (bei Kamenz), doch möchte ich bemerken, dafs ich gerade bei dieser Ente oft die Neigung beobachten konnte, in der Brutzeit auf Teichen zu erscheinen, wo sie sich nicht fortpflanzt (z. B. Grofshartmannsdorf), sodafs vorstehende Notizen nur bedingten Wert haben und sich kein Beobachter verleiten lassen sollte, aus blofsen Beobachtungen während der Brutzeit den Nachweis des Brütens herzuleiten. Ihre Ankunft beobachtete ich zeitigstens am 10. März, die letzten Herbstwanderer Mitte November. Kirbach nennt sie fälschlich ,eine charakteristische Erscheinung unter den Wintergästen" des Elbflusses bei Meifsen.

\section{Anas boschas L.}

Anas boschas Linn. - Meyer und Helm, Verz. Vög. Kgr. Sachs. p. 119.

Wilde Ente. Als verbreitetste und an allen Orten häufigste Brutente bewohnt die Stockente nicht nur die grofsen Teichreviere, sondern auch solche kleinerer Ausdehnung bis herab zum verschilften Dorftümpel und unsere Flufsläufe, an deren bewaldeten und bebuschten Uferböschungen sie oft genug auch brütet. Im Gebirge steigt sie nach Berge im allgemeinen nicht bis $600 \mathrm{~m}$ Höhe, was sich, bis auf Ausnahmen, auch mit meinen Erfahrungen deckt. Bläser und Gottsmann stellten sie bei Tobertitz i. Vogtl.) in $500 \mathrm{~m}$, ich auf dem Filzteich $(540 \mathrm{~m})$ und auf den Grofshartmannsdorfer Teichen in ebenfalls $500 \mathrm{~m}$ Höhe als Brutvogel fest, wo sie auch, genau wie in den niederen Lagen, überwintert. Fast alljährlich auch 1914, brütete ein Paar auch auf den Galgenteichen bei Altenberg $(780 \mathrm{~m})$.

\section{Anas strepera L.}

Anas strepera Linn. - Meyer und Helm, Verz. Vög. Kgr. Sachs. p. 119.

Nach den früher über das Brutvorkommen der Mittelente vorliegenden Notizen kannten sie Meyer und Helm anfangs der neunziger Jahre nur als sehr vereinzelten Brutvogel, was für die Gegenwart jedoch nicht mehr gilt. Sie kommt vielmehr in fast allen grofsen Teichgebieten der Niederung als solcher vor und kommt an Häufigkeit wohl der Löffelente gleich. So brütet sie regelmäfsig und, wie Baer nach liramer berichtet, nicht selten 
auf den Teichen des Lausitzer Tieflandes, wo sie Hantzsch nicht gerade häufig, aber auch nicht selten bei Königswartha, ständig zwischen diesem Ort und Caminau fand und auch ich sie Juni 1914 beobachtete. Auf dem Horstsee bei Wermsdorf traf ich jeden Sommer einige Paare an, überraschte am 5. VII. 1908 auch ein $\&$ mit 11 pulli. Den Moritzburger Teichen fehlt sie nach Helm, auf denen von Rohrbach tut sie das nur zeitweilig, denn Hennicke kannte sie als Brutvogel von dort, und Hesse konstatierte 1905 auch ein Brutpaar daselbst. Die Teiche zwischen Frohburg und Eschefeld, auf denen sie nach J. Kratzsch seit 1868 eingewandert ist, gehören ebenfalls zu ibren Brutstätten, wie die Angaben verschiedener Beobachter, Nestfunde von Zimmermann und Weifsmantel und eigene Studien verbürgen. Im Grofshartmannsdorfer Gebiet, dessen Vegetationsverhältnisse ihr wohl nicht zusagen, brütet sie nicht, gleichwohl zeigte sich 17. VI. $19131 \sigma^{7}$ hier. Auf dem Zuge erscheint sie natürlich, wie auch die anderen Brutenten, nicht nur auf den Brutteichen häufiger, sondern auch andernorts, zuweilen sogar auf den Flufsläufen. Der Frühjahrszug setzt in der Regel im letzten Drittel des März ein, und der Herbstzug verlöscht im Oktober, doch kommen auch hier Regelwidrigkeiten vor. So schofs nach Wichtrich Hülsmann 1 Stück schon am 5. I. 1910 bei Nepperwitz (bei Wurzen), und Helm beobachtete $2 \sigma^{\nearrow}, 1 \&$ am 12. II. 1899 auf dem Ziegelteich bei Frohburg.

\section{Anas penelope L.}

Anas penelope Linn. - Meyer und Helm, Verz. Vög. Kgr. Sachs. p. 120.

Die Pfeifente berührt das Königreich Sachsen regelmäfsig als Durchzugsvogel von März bis Mai und September bis November und wird dann auf ihren Rastplätzen nicht selten in gröfseren Ansammlungen beobachtet; Helm und Weifsmantel sahen auf den Frohburger Teichen mebrfach Hunderte dieser Vögel. Wie schon gesagt, verzögert sich ihr Durchzug nicht selten bis in den Mai hinein, in welchem Monat sie von Helm, Berge, Hesse und auch von mir wiederholt beobachtet wurde. Helm versicherte mir sogar, ein Pärchen im Mai bei der Begattung auf den Frohburger Teichen belauscht zu haben. Diese Gewohnheit, sich noch öfter als die anderen Zugenten an den Rastorten bis in den Mai zu verweilen, hat wohl veranlafst, dafs Hennicke in ihr einen seltenen Brutvogel der Rohrbacher T'eiche sah. Auch Fritzsche hielt sie für einen Brutvogel der Moritzburger Teiche, weil sie im Sommer 1879 in ziemlicher Anzahl daselbst erlegt wurde. Obwohl ein Brüten dieser Ente in Sachsen nicht nur nicht ausgeschlossen, sondern wahrscheinlich ist, scheint man doch in beiden Fällen nicht die zum sicheren Nachweis des Brutvorkommens nötigen Beobachtungen von Vögeln am Nest oder mit Jungen gemacht zu haben, weshalb ein vollgültiger Nachweis des Brütens im Gebiet bis heute fehlt. 
Daran ändert auch ein Erpel nichts, der am 31. VIII. 1884 auf dem Brösaer Teich (bei Guttau i. d. Lausitz ${ }^{1}$ ) erlegt ist, den die Sammlung der Forstakademie Tharandt besitzt und der möglicherweise bei uns ,übersommert" hat. Einzeln kommen Pfeifenten auch in den Wintermonaten vor.

\section{Anas acuta L.}

Anas acuta Linn. - Meyer und Helm, Verz. Vög. Kgr. Sachs. p. 119.

Fasanente. Die Spiefsente hat in ihrem Vorkommen manches mit der vorhergehenden gemein, erscheint wie diese regelmälsig März - April und Oktober - November auf dem Zuge, ist aber stets erheblich seltener. Überwinterungen sind ebenfalls vereinzelt $\mathrm{zu}$ verzeichnen; H. Naumann erhielt sie 12. XII. 1891 aus Niedergurig (Laus.), und Hülsmann beobachtete 4 St. im Januar 1888 bei Wurzen auf der Mulde. Sie hat auch, wenngleich als Seltenheit, im Gebiet gebrütet, denn nach Baer beobachtete Kramer am 11. VII. 1893 eine Brut von 6 Dunenjungen bei Königswartha, und v. Vietinghoff nimmt, anscheinend ohne Kenntnis jener Baer'schen Notiz, ebenfalls an, sie sei Brutvogel, weil sie im Sommer zuweilen bei Königswartha erlegt werde. Ein auf dem Zug verspätetes $\sigma^{x}$ beobachtete ich 5. V. 1907 auf dem Horstsee bei Wermsdorf.

\section{Anas querquedula L.}

Anas querquedula Linn. - Meyer und Helm, Verz. Vög. Kgr. Sachs. p. 120.

Schnärrente. Die Knäckente ist ein zahlreicher Brutvogel fast aller reichlich bewachsenen Gewässer der Niederung, der nur die dürftig bestandenen Teiche, wie die Waldteiche bei Wermsdorf, und solche höherer Lagen meidet. Auf den Teichen von Grofshennersdorf (bei Ostritz, $280 \mathrm{~m}$ ) hat sie nach Kramer öfter gebrütet; höher hinauf habe ich sie nicht feststellen können. Im März ist ihr Erscheinen zu erwarten; im Oktober geht sie.

\section{Anas crecca L.}

Anas crecca Linn. - Meyer und Helm, Verz. Vög. Kgr. Sachs, p. 120 .

Die Krickente ist im Niederlandgebiet als Brutvogel ebenfalls nicht selten, doch tritt sie gegen vorige etwas zurück, fehlt aber trotzdem wohl keinem gröfseren Teichgelände völlig. Dafür ist sie auf den unbedeutenden Teichen des Hügellandes verbreiteter und geht im Gebirge hoch hinauf, sodafs sie von

1) Für die benachbarte pre u fs. Oberlausitz ist das Brüten von Anas penelope nachgewiesen. 
allen Brutenten die gröfste Verbreitung aufweist. Im Niederland hält sie sich gern zu den seichten Teichpartien und ihren sumpfigen Ausläufern sowie den Dickichten von Sparganium erectum; im Gebirge dagegen sind für ihre Aufenthalte bezeichnend die mit Wasser gefüllten Ausstiche und „Augen" der erzgebirgischen Hochmoore und die moorigen Komplexe der Umgebung dortiger Teiche. An diesen Orten fehlt Anas boschas entweder oder ist seltener als sie, wie z. B. in den Torfstichen beim Grofshartmannsdorfer Grofsen Teich $(500 \mathrm{~m})$. Nach Berge brütet sie auf dem "Filz" bei Schneeberg $(540 \mathrm{~m})$, und sogar auf dem hart jenseits der Grenze liegenden Gottesgaber Moor $(1050 \mathrm{~m})$ wurden nach diesem Schriftsteller schon Bruten festgestellt. Als Zugvogel fehlt sie natürlich keinem Teichgebiet, kommt im März, gelegentlich auch früher an und geht im November, überwintert zuweilen auch in den niederen Lagen.

\section{Chenalopex aegyptiacus (L.).}

Nach Hülsmann sind im August 1887 einige Nilgänse in „diverser Umgebung“ von Wurzen erlegt worden. Offenbar meint er damit jene Vögel, die bei Gotha (bei Eilenburg), jenseits der Grenze erbeutet sind und über welche H. Hertwig (Orn. Monatsschr. 13. Jg. [1888] p. 167) berichtet.

\section{Tadorna tadorna (L.).}

Tardorna cornuta (Gm.). - Meyer und Helm, Verz. Vög. Kgr. Sachs. p. 119.

Die Brandgans ist für Sachsen als seltener Gast zu betrachten. Da Gänse vielfach gefangen gehalten werden, dürften sich bei dieser und den folgenden Arten da und dort unter den erwähnten Vögeln auch aus der Gefangenschaft entwichene befinden.

Nach Reichenbach kam sie schon früher bisweilen zu uns, so wurde im April 1834 eine bei Breitenfeld (bei Leipzig) geschossen. Das Zoologische Museum Dresden besitzt 1 ㅇ, am 16. X. 1897 bei Dippelsdorf geschossen. Hennicke und später auch Hesse erwähnen ihr Vorkommen auf den Rohrbacher Teichen, doch geht aus ihren Mitteilungen hervor, dafs sie die Gans nicht selbst beobachtet haben. Zugetragenen Nachrichten über das Vorkommen von Brandgänsen (Brandenten) aber mufs mit äufserster Vorsicht entgegengetreten werden, weil im Volksmund da und dort auch die Tafelente „Brandente“ genannt wird und dies in folgenden Fällen wahrscheinlich auch zu solchen Verwechselungen Anlafs gegeben hat: Forstmeister v. Brandenstein bemerkt in den Jahresberichten, sie werde bei Hubertusburg (bei Wermsdorf) vorübergehend Sommer und Herbst beobachtet, und Kluge verzeichnet sie ohne weitere Bemerkung für Uhyst (am Taucher). Die Angabe Kirbachs, sie komme ,im Herbst zu 
uns" (das ist in die Gegend von Meifsen), ist viel zu unbestimmt, um als Nachweis zu gelten.

\section{Casarca casarca (L.).}

Tadorna casarca (Linn.). - Meyer und Helm, Verz. Vög. Kgr. Sachs. p. 119.

Zu Reichenbachs Zeiten besafs das Zoolog. Museum Dresden 1 Exemplar von den Moritzburger Teichen. Ich kenne keine weiteren sächsischen Nachweise dieses sehr seltenen Gastes, denn alle in neuerer Zeit erlegten Rostenten erwiesen sich als Flüchtlinge aus Zoolog. Gärten etc., so z. B. 21905 oder 1906 bei Grofsenhain geschossene.

\section{Anser anser (L.).}

Anser cinereus Meyer. - Meyer und Helm, Verz. Vög. Kgr. Sachs. p. 118.

Trotzdem den meisten sächsischen Beobachtern das Unterscheiden von Grau- und Saatgänsen in der Freiheit, das sich auf geringfügige und bei der seltenen Gelegenheit solcher Beobachtungen trügerische Kennzeichen stützt, nicht geläufig gewesen sein mag, hat man alle vorgekommenen grauen Gänse bald zur Saatgans, hauptsächlich aber zur Graugans gestellt. In diesen Fehler verfallen hauptsächlich die Meyer und Helm'schen Berichterstatter. Die auf diese Weise gewonnenen Daten über das Auftreten und den Zug dieser Arten sind infolgedessen von nur bedingter Brauchbarkeit. Ich mufs gestehen, dafs mir nur von wenigen Arten ein so dürftiges authentisches Datenmaterial vorgelegen hat als gerade von der Graugans. Doch läfst sich sagen, dafs sie für unser Sachsen ein ziemlich seltener Durchzügler ist, der nach Berge bis in den Anfang des neunzehnten Jahrhunderts auch gebrütet hat. Nach Peschel geschah dies noch um 1825 in den Rödersümpfen. Obwohl z. B. bei Zwickau nach Berge "öfters erlegt" ist mir doch kein Belegstück vorgekommen. Der Durchzug geht Februar und März, August und September, nach Berge selbst bis Dezember von sich. Popelka will sogar am 21. V. 1887 noch eine bei Neugersdorf (bei Löbau) gesehen haben.

\section{Anser fabalis (Lath.).}

Anser segetum Meyer. - Meyer und Helm, Verz. Vög. Kgr. Sachs. p. 118.

Schneegans, Wilde Gans. Die Saatgans erscheint im Gebiet als ziemlich seltener Durchzügler und überwintert in geeigneten Gegenden in mehr oder minder grofser Zahl. Als bevorzugte Überwinterungsgebiete sind die schneearmen und ebenen Gegenden am Unterlauf von Röder, Elbe und Mulde bekannt. Peschel spricht 
1885 von Tausenden ${ }^{1}$ ), die hier den Winter verbringen. Der Durchzug setzt im September ein und endet im März. Ein Belegstück besitzt die Sammlung der Forstakademie Tharandt: \$, 17. XII. 1887, Pillnitz (bei Dresden).

Was das Vorkommen der noch immer umstrittenen Ackergans (Anser fabalis arvensis Br.) angeht, so liegen weder Belege noch zuverlässige Nachrichten vor. Bei der Neubearbeitung des Naumann stellt zwar Helm zwei von Hülsmann veröffentlichte Vorkommnisse aus der Wurzener Gegend zur Ackergans, doch sagt Hülsmann in der einen Originalnotiz ausdrücklich, dafs es sich um Exemplare der kleineren Subspezies, also um $A$. fabalis, gehandelt habe, und auch die andere lärst Zweifel offen.

\section{Anser albifrons (Scop.).}

Anser albifrons Bechst. - Meyer und Helm, Verz. Vög. Kgr. Sachs. p. 118.

Sehr seltener Durchzügler. Mosch erwähnt die Bläfsgans für Reinhardtsdorf (Sächs. Schweiz), und Reichenbach erhielt am 30. IX. 1835 vom Forstmeister v. Haake in Schandau (ebenfalls in der Sächs. Schweiz) 2 dort erlegte, im ersten Jahre stehende Vögel, denen die Blässe noch fehlte.

\section{Anser erythropus (L.).}

Die Zwerggans ist einmal bisher als sehr seltener Gast für unser Gebiet festgestellt. Die Sammlung der Forstakademie Tharandt besitzt ein am 17. XI. 1888 auf Reinhardtsdorfer Revier (Sächs. Schweiz) verendet aufgefundenes junges $\&$.

\section{Anser hyperboreus Pall.}

Einmal beobachteter sehr seltener Gast. Wichtrich beobachtete am 10. XII. 1902 bei Eutritzsch (bei Leipzig) 4 überhinfliegende Schneegänse, sah deutlich „die schwärzliche Einrahmung auf der Unterseite der Flügel" und fand die Flügelspitzen besonders schwarz. Ich halte bei dieser Art eine Verwechselung für ausgeschlossen.

\section{Cygnopsis cygnoides (L.).}

Päfsler führt diese sibirische Gans für Meerane unter denjenigen Vögeln auf, „welche auf ihrer Wanderung und ihren Streifzügen" vorkommen. Er hat sich hierzu offenbar durch ein der Gefangenschaft entflogenes Stück verleiten lassen.

1) P. nennt diese Gănse allerdings „Graugănse“, gemeint sind mit diesen Wintervogeln nur Saatgănse. 


\section{Branta bernicla (L.).}

Bernicla torquata Bechst. - Meyer und Helm, Verz. Vög. Kgr. Sachs. p. 117.

Die Ringelgans kommt nach Sachsen als seltener Durchzugsund gelegentlicher Wintervogel. Reichenbach erhielt eine frisch erlegte am 19. X. 1837 aus Postelwitz (bei Schandau), Dehne am 10. XII. 18522 Stück, die auf der Elbe bei Kötzschenbroda erlegt waren. Schon einige Jahre vorher hatte letzterer Schriftsteller 3 solche Gänse über das Lö fsnitztal bei Dresden ziehen sehen. Held besafs in seiner Sammlung ein 오, 1. III. $1883 \mathrm{im}$ Königsholz bei Oderwitz geschossen, in Museum des „Gebirgsvereins für die Sächs. Schweiz" in Pirna steht eine von Wünsche präparierte Ringelgans, am 21. II. 1889 bei Schandau erbeutet, und die Sammlung der Forstakademie Tharandt enthält ein jüngeres Stück aus Grofsenhain. Aufserdem erwähnt Strohbach ein weiteres Exemplar von Anfang Januar 1889 aus Gablenz (bei Chemnitz), und Helm beobachtete am 20. III. 1898 l auf den Frohburger Teichen.

\section{Cygnus olor (Gm.).}

Cygnus olor (Gm.) - Meyer und Helm, Verz. Vög. Kgr. Sachs. p. 118.

Was ich bei der Brandgans bezüglich des Vorkommens von der Gefangenschaft entflohenen Gänsen sagte, gilt in fast noch höherem Mafse vom Höckerschwan. Zweifellos setzt sich die Mehrzahl der im Gebiet geschossenen Schwäne aus solchen Flüchtlingen zusammen. Insbesondere werden auch die, welche da und dort den Sommer verbrachten, halbzahm gehaltene und entwischte Vögel sein. Diese Art ist also nur als seltener Durchzugsvogel anzusehen. Hennicke berichtet von 2 Stück, die 1893 von Mai bis September auf den Rohrbacher Teichen anzutreffen waren, und Neumann und Grünewald beobachteten diese Art am 21. V. 1887 auf dem Spitalteich, zwei Tage später in den Röderwiesen bei Grofsenhain. Nach Koepert beobachtete auch Helm ein Paar im Oktober 1895 auf den Teichen bei Frohburg. Das Zool. Museum Dresden besitzt Ơㅇ, am 6. XII. 1896 bei Kaditz (bei Dresden) auf der Elbe geschossen. Berge erwähnt einen Jungen, am 29. XII. 1898 auf der Mulde bei Schindmaas (bei Glauchau) erbeutet, und vom gleichen Flufs stammt das Exemplar der Realschule Rochlitz, das bei Stöbnig (bei Rochlitz) zu Ende der 1890 er Jahre erlegt ist.

\section{Cygnus cygnus (L.).}

Cygnus musicus Bechst. - Meyer und Helm, Verz. Vög. Kgr. Sachs. p. 118.

Seltener Durchzügler, gelegentlich auch i $\mathrm{m}$ W in t e r vorkommend. Das Dresdener Museum besafs zu Reichenbachs Zeit 
sächsische Singschwäne, und in der Sammlung des Zoolog. Instituts Leipzig steht nach Hesse heute noch ein Alter aus Möckern (bei Leipzig), 1809 erlegt. Nach Schicker hielten sich 18783 Stück einige Tage bei Friesen (bei Reichenbach) auf; nach Stiehler zeigte sich Anfang 1887 einer auf der zugefrorenen Elbe bei Pirna; nach Berge schofs man einen Jungen im September 1885 auf dem Zwickauer Schwanteich; nach Etzold wurde am 18. I. 1891 einer von vieren auf der Elster bei Groitzsch geschossen; nach Marx sen. geschah dasselbe mit 2 anderen Mitte Januar 1892 auf der Elbe bei Riesa; nach Hennicke wurde einer auf den Rohrbacher Teichen gefangen; Strohbach beobachtete 21. und 22. XI. 1889 durchziehende bei Chemnitz, Helm 3 Stück Ende März 1893 auf dem Frauenteich bei Moritzburg, und Held kannte einen, der im Herbst 1857 bei Türchau (bei Zittau) erlegt worden war.

\section{Cygnus bewicki Yarr.}

Cygnus minor Pall. - Meyer und Helm, Verz. Vög. Kgr. Sachs. p. 118. Einmal nachgewiesener sehr seltener Gast.

Nach J. Fr. Naumann wurde ein Zwergschwan im Januar 1823 aus einem 'Trupp von 3 Stück bei Möckern (bei Leipzig) geschossen. Reichenbach erwähnt diesen Fall ebenfalls, sagt aber fälschlich „1828“.

\section{Haematopus ostralegus L.}

Haematopus ostralegus Linn. - Meyer und Helm, Verz. Vög. Kgr. Sachs. p. 111.

Der Austernfischer ist sehr seltener Gast im Gebiet. Reichenbach sagt er sei "selten bei uns" und nennt als Fundort Moritzburg. Eimert führt ihn mit dem Vermerk "sehr selten“" für Zittau auf, enthält sich jedoch näherer Angaben. Im Zool. Museum Dresden steht ein $\sigma^{\top}$ aus Wildenhain (bei Grofsenhain) vom 31. I. 1908.

\section{Arenaria interpres (L.).}

Der Steinwälzer ist ebenfalls sehr seltener Gast, denn von einem, wenn auch noch so geringem Durchzug kann wohl bei dieser und der vorigen Art nicht gesprochen werden. Wichtrich beobachtete am 3. und 4. IX. 19101 jüngeres Exemplar am Ziegelteich bei Frohburg und am 21. V. 19111 altes $\sigma^{\top}$ an der Mulde bei Nischwitz (bei Wurzen). Auch ich sah am 21. VIII. $1911 \mathrm{am}$ Gr. Teich bei Grofshartmannsdorf (bei Freiberg) 1 anscheinend älteres Exemplar unter so günstigen Umständen, dafs ein Irrtum ausgeschlossen ist.

\section{Cursorius gallicus (Gm.).}

Cursorius europaeus Lath. - Meyer und Helm, Verz. Vög. Kgr. Sachs. p. 110. 
Auch der Rennvogel ist als sehr seltener Gast nachgewiesen. Nach Helm wurde nämlich Ende August 1889 ein junger Vogel bei Potschappel (bei Dresden) und im September 1891 ein Alter in der Gegend von Grofsenhain erbeutet. Über ihren Verbleib ist leider nichts bekannt.

\section{Glareola fusca (L.).}

Mosch versichert, bei der Zschiepmühle bei Schönau (Sächs. Schweiz) sei als grofse Seltenheit „Glareola torquata" geschossen worden. Bei der schon erwähnten und im ersten Teil dieser Arbeit auch ausführlicher bewiesenen Unzuverlässigkeit Moschs ist die Verwechselung mit einer anderen Art nicht ausgeschlossen.

\section{Squatarola squatarola (L.).}

Charadrius squatarola (Linn.). - Meyer und Helm, Verz. Vög. Kgr. Sachs. p. 110.

Der Kiebitzregenpfeifer berührt das Königreich als seltener Durchzügler. J. G. Neumann bezeichnet ihn als bei Hirschfelde (bei Zittau) erlegt, und nach Koepert ist er auch bei Eschefeld (bei Frohburg) von Porzig geschossen worden. Ruhsam besafs in seiner Sammlung 1 St., 28. X. 1880 bei Geyersdorf (bei Annaberg) erlegt, Berge kannte einen, der im September 1903 bei Zwickau erbeutet war, und Jacobi erwähnt 1 junges $\$$, Mitte September 1887 aus der Gegend von Leipzig. Helm hat ihn wiederholt bemerkt, so am 21. IX. 1889 2, am 23. IX. 18894 im Gr. Gehege, 6. X. 1889 l am Frauenteich und 27. IX. 1891 2 am Dippelsdorfer Teich bei Moritzburg. Nach Jakobi v. Wangelin fand Helm auch noch am 10. XI. 18954 bei Frohburg. Hesse fand ihn auch im Frühling: 2 Stück am 16. IV. 1904 bei Gundorf (bei Leipzig).

\section{Charadrius apricarius L.}

Charadrius pluvialis Linn. - Meyer und Helm, Verz. Vög. Kgr. Sachs. p. 110.

Der Goldregenpfeifer kommt nach Sachsen als ziemlich seltener Durchzügler in den Monaten März, September, Oktober, seltener November. Hesse beobachtete sogar noch am 10. XII. 190611 St. bei Rohrbach und Rey erhielt ihn wieder am 2. II. 1907 aus Lauer (bei Leipzig).

\section{Charadrius morinellus L.}

Eudromias morinellus Linn. - Meyer und Helm, Verz. Vög. Kgr. Sachs. p. 110.

Der Mornell-Regenpfeifer konnte als sehr seltener Gast während der Zugzeiten immerhin einige Male nachgewiesen 
werden. J. G. Neumann bucht ihn als bei Hirschfelde (bei Zittau) vorgekommen. Nach Reichenbach erhielt das Dresdener Museum ein zerschossenes Stück, das am 26. IX. 1835 aus einem Flug vom 10 Stück bei Kesselsdorf (bei Dresden) erlegt war. Held besals in seiner Sammlung 1 웅 Herbst 1866, aus Eckartsberg (bei Zittau). Nach Berge wurde April 1889 einer verendet bei Mylau i. V. gefunden, Jacobi [oder Lohse] tut eines jüngeren Erwähnung, der 10. X. 1889 an der Mulde nahe der preufsischen Grenze erlegt wurde, und nach Geisler zeigten sich am 25. X. 18942 auf Jesauer Flur (bei Bautzen).

\section{Charadricus hiaticula L.}

Aegialites hiaticula Linn. - Meyer und Helm, Nachtrag zum Verz. Vög. Kgr. Sachs. [1896] p. 2.

Der Sandregenpfeifer kommt gelegentlich des Zuges als seltener Passant durch unser Gebiet. Ich kenne folgende Fälle seines Auftretens: Mosch zählt ihn für Sachsen ohne nähere Bemerkungen auf, uud E. J. J. Meyer erwähnt ihn in gleicher Form für Dresden. Berge gedenkt seiner als bei Zwickau erlegt. Nach Koepert wurde er von Schach auch bei Rufsdorf (bei Crimmitschau) geschossen. Schwarze beobachtete 20. VIII. 18942 bei Kaditz (bei Dresden) und schofs 2 jetzt im Zoolog. Museum Dresden befindliche $\sigma^{x}$ am 24. und 25. IX. 1894 auf den Elbhegern bei Dresden. Aufser diesen liegen im Museum noch $1 \sigma^{x}, 2$ 우 vom 3. X. 1899 aus Moritzburg. Helm beobachtete diesen Regenpfeifer wiederholt, so Anfang Oktober 1899 am Frauenteich bei Moritzburg 6, am Dippelsdorfer Teich sogar 20 Stück, am 6. IV. 1902 einen bei Frohburg, am 11. V. 1902 ebenda 4 und am 8. und 22. VI. desselben Jahres nochmals je einen. Ein ähnlich gelegentliches Auftreten während der Brutzeit mag Päfsler zu der irrigen Annahme verleitet haben, er brüte bei Meerane. 


\section{$2 \mathrm{BHL}$ Biodiversity Heritage Library}

1916. "Die geographische Verbreitung der Vögel im Königreich Sachsen. Allgemeiner Teil." Journal fu

r Ornithologie 64, 196-228. https://doi.org/10.1007/bf02250520.

View This Item Online: https://www.biodiversitylibrary.org/item/43088

DOI: https://doi.org/10.1007/bf02250520

Permalink: https://www.biodiversitylibrary.org/partpdf/142436

\section{Holding Institution}

MBLWHOI Library

\section{Sponsored by}

MBLWHOI Library

\section{Copyright \& Reuse}

Copyright Status: No known copyright restrictions as determined by scanning institution.

This document was created from content at the Biodiversity Heritage Library, the world's largest open access digital library for biodiversity literature and archives. Visit BHL at https://www.biodiversitylibrary.org. 